\title{
Universal Rank-Order Transform to Extract Signals from Noisy Data
}

\author{
Glenn Ierley ${ }^{*}$ \\ Mathematics Department, Michigan Technological University 1400 Townsend Drive, \\ Houghton, Michigan 49931, USA \\ and Scripps Institution of Oceanography, UC San Diego, 9500 Gilman Drive, \\ La Jolla, California 92093-0225, USA \\ Alex Kostinski ${ }^{\dagger}$ \\ Physics Department, Michigan Technological University 1400 Townsend Drive, \\ Houghton, Michigan 49931, USA
}

(Received 28 November 2018; revised manuscript received 21 March 2019; published 3 September 2019)

\begin{abstract}
We introduce an ordinate method for noisy data analysis, based solely on rank information and thus insensitive to outliers. The method is nonparametric and objective, and the required data processing is parsimonious. The main ingredients include a rank-order data matrix and its transform to a stable form, which provide linear trends in excellent agreement with least squares regression, despite the loss of magnitude information. A group symmetry orthogonal decomposition of the 2D rank-order transform for iid (white) noise is further ordered by principal component analysis. This two-step procedure provides a noise "etalon" used to characterize arbitrary stationary stochastic processes. The method readily distinguishes both the Ornstein-Uhlenbeck process and chaos generated by the logistic map from white noise. Ranking within randomness differs fundamentally from that in deterministic chaos and signals, thus forming the basis for signal detection. To further illustrate the breadth of applications, we apply this ordinate method to the canonical nonlinear parameter estimation problem of two-species radioactive decay, outperforming specialpurpose least squares software. We demonstrate that the method excels when extracting trends in heavy-tailed noise and, unlike the Thiele-Sen estimator, is not limited to linear regression. A simple expression is given that yields a close approximation for signal extraction of an underlying, generally nonlinear signal.

DOI: 10.1103/PhysRevX.9.031039

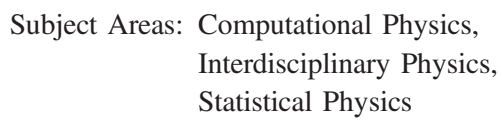

\section{INTRODUCTION}

We report on a discovery of a rank-based method that appears remarkably versatile and robust with respect to the nature of noise because the method is ordinal, nonparametric, and therefore distribution independent. Throughout the paper, the performance of the method is compared to leading nonparametric tests and software, using real as well as synthetic data, where exact results are known. As new results abound (the most important ones appear in Sec. V and after), we begin with the slightly unconventional device of detailed overview to orient the reader.

\footnotetext{
*Emeritus, Scripps Institution of Oceanography. grierley@ ucsd.edu

tkostinsk@mtu.edu

Published by the American Physical Society under the terms of the Creative Commons Attribution 4.0 International license. Further distribution of this work must maintain attribution to the author(s) and the published article's title, journal citation, and DOI.
}

In Sec. II, we introduce and motivate the initial construction of our method (dubbed the $Q$ transform) in a simple setting: We begin by solving for the long-term warming trend buried in a fluctuating time series of daily low temperature. The same quantity was later identified as a diagnostic for signal detection and is simultaneously used here for signal extraction by means of parameter estimation (here, the slope). Agreement with the least squares method is excellent. This agreement is quite surprising, given that the method retains no magnitude information whatsoever, only rank. This is a setting with few outliers, where the two approaches generally agree.

In Sec. III, we propose a continuous approximation for $Q$, in terms of which one can understand $Q$ as a simple 2D integral transform. This formulation facilitates an accurate approximation of various basic results (Figs. 2, 3, 7, and 17) with algebraic forms that are more transparent in meaning than the equivalent discrete forms.

In Sec. IV, we introduce two statistical metrics used for confidence tests, characterize their distributions, and give 
an asymptotic approximation for the scaling of each. The case of correlated noise is also considered.

In Sec. V, we give a universal representation of the $Q$ transform for all distributions of identically and independently distributed (iid) (white) noise. The key is a five-term exact orthogonal decomposition based on planar group character, applied to all realizations of $Q$ in an ensemble. Principal component analysis (PCA) is used on each of the resulting group ensembles. The lifting of the original $1 \mathrm{D}$ time series to the 2D rank-order space of $Q$ - "order" here is taken as timelike, but generally representing any serial, independent variable - establishes a link between $Q$ modes and corresponding ordered patterns of sample variability in mean and variance. As a consequence, $Q$-based slope estimates from Sec. II for long-term trends are unaffected by trends in variance. These ideas are further developed in Sec. VI, where a new metric is developed for characterizing stochastic processes, offering a prejudice-free means of selecting a model for experimental data.

In Sec. VII, we address a detection problem where the signal is a chaotic series generated by the logistic map. Our method, which makes no assumptions about the functional form of the underlying signal, readily detects the presence of chaotic signals, whether alone or in combination with white noise.

In Sec. VIII, we consider the canonical nonlinear parameter estimation problem for noisy two-species radioactive decay (see Chap. 8 in Ref. [1]). In this problem of quantitative signal extraction, our method outperforms special-purpose least squares software by stably retrieving both decay rates.

In Sec. IX, we introduce a heuristic approximation for extracting a complex signal up to within a linear rescaling by simple differentiation of the transformed field.

In Sec. X, two data sets with distributions of infinite mean and variance noise are explored. For such distributions, the Theil-Sen nonparametric method is commonly used, but it is limited to linear regression. Our transform also succeeds for the linear problem but extends to arbitrary functional forms and multilinear settings as well.

In Sec. XI, we close with an extension of the method to unequally spaced time series. We develop the theoretical basis for error analysis and apply it to linear regression, hence accounting for the otherwise enigmatic agreement of the linear fits exhibited in Sec. II.

\section{A. Signal detection}

To place the $Q$ transform within the existing literature on time-series analysis, first consider signal detection where statistical signal processing is, perhaps, the natural setting. Here, one devises a test statistic (e.g., a local estimate of power) and selects an operating threshold [2,3] to decide whether a signal is present. Performance as judged by misses and false alarms is often characterized with a receiver operating characteristic curve. If used in the time domain, most such detectors are local; they use a single realization consisting of a short segment of the signal to evaluate the test statistic. The resulting sequence of such statistics for the entire time series identifies intervals where signal is likely present.

In contrast to such local cardinal measures, the method proposed here is both global and ordinal. By global, we mean that evaluation of $Q$ relies on a significant number of trials to accumulate sufficient statistics about the parent noise distribution. This global approach performs well for detection at a poor signal-to-noise-ratio (SNR) when local methods would fail. The drawback is that $Q$ obtained from a single trial partitioning may be prohibitively large.(See Sec. II) To appreciate the novelty, consider, for example, that the three-volume set [2] on statistical signal processing does not suggest a single ordinal method.

\section{B. Signal extraction}

The subsequent problem of signal extraction is often accomplished by some variant of a least squares minimization, and a vast literature supports this approach. For example, when errors are iid Gaussian random variables, ordinary least squares is the maximum likelihood estimator (e.g., see Refs. $[1,4])$. However, nonstationary variance is ubiquitous in data analysis as is lack of independence. These complications could be addressed with generalized least squares using a weight matrix equal to the inverse of the covariance matrix, $\Omega$, when the covariance of the fluctuations is known. In practice, $\Omega$ must be estimated. For this "feasible generalized least squares," it is difficult to assess the effect of error with empirical weights. Correlated nonstationary noise is often heavy tailed (e.g., see Ref. [5] for numerous examples in atomic physics), and outliers are then a serious problem for least squares. Rank-based methods need no empirical weights for such complications. Two-species radioactive decay is a case where the least squares error itself-nonlinear in the parameters-may fail as a penalty function, while our rank-based measure proves robust.

For parameter estimation, one chooses a representation for the solution, either specific to the application, as with exponential decay, or a generic form such as a polynomial expansion. The coefficients in the functional form are determined by a minimization procedure.

For nonparametric signal extraction, we make no assumption about form apart from spectral separation. The natural comparison for a deterministic signal buried in noise is a moving average convolution, with the stencil of weights ranging from a simple boxcar to a precisely designed filter for impulse response. Such filters are applied to single realizations, whereas $Q$ benefits from multiple realizations.

In summary, in the realms of both detection and extraction, to the best of our knowledge, there are no methods that are rank based, nonparametric, and global. 


\section{DESCRIPTION OF THE $Q$ TRANSFORM AND TREND EXTRACTION}

We choose climate as a setting to initially motivate and illustrate the method, but several other contexts will be provided throughout the paper. Nonparametric statistics have been used in climate physics; e.g., record-breaking statistics have been employed to infer a variety of trends from temperature time series [6-9]. Such nonparametric and distribution-free methods are, indeed, an alternative to the various least squares methods. However, to the best of our knowledge, up to now only record lows and record highs have been used in the climate context (e.g., Ref. [10]). Here, we are guided by the simple thought that the entire rank information and not just its first and last elements, ought to be used in nonparametric analyses, and our results buttress this claim. Throughout this paper, the $i$ th entry in a time series, $x_{i}$, is assigned rank $r$ if it is the $r$ th lowest value of the entire sequence when sorted by magnitude. For example, the high or low daily temperature at a particular location, $T=T(t)$, is sorted and ranked below, and we track the year of origin (order, $t$ ), hence the "rank-order" in the title. We note in passing that rank is not always uniquely defined as ties occur [11]. To circumvent this problem, we either assign fractional rank or add white noise. In this paper, we examine data sets of daily high temperatures from the Global Historical Climatology Network (GHCN) and raw monthly mean temperatures from the Berkeley Earth repository.

As a specific example, consider the GHCN weather station SZ000009480 (Lugano, Switzerland). Color is used in Fig. 1(a) to display daily high-temperature values as a day (row) and year (column) matrix. The seasonal variability is apparent; e.g., almost everything is red around day 180 (summer). In Fig. 1(b), we display the same data but with daily rank recorded in rows: All magnitude information has been discarded, and all data are now integer valued. The appearance is fine grained, reminiscent of "salt-andpepper" noise. The central finding of this paper is that the trend information content of Figs. 1(a) and 1(b) is almost identical (for a large data set), despite the total loss of magnitude data. This finding is appealing, as ranking is affected neither by outliers nor any monotonic transformation of temperature data, e.g., a logarithm [12], nor by occasional gaps in data as shown below. To introduce the approach, we begin by repackaging the day and year rank matrix data.

Disregarding the dependence (for the moment), let us view the daily temperature values in Fig. 1(b) as independent random trials, indexed by year. For example, among the 365 trials during year 1951, nine record-low $(r=1)$ values occurred, that is, lower than any of the 63 subsequent values (1952-2014) for that day. Given the independent-trials perspective, the essential information can be distilled to just three numbers: Only the year, the rank, and the "population" of that rank need to be preserved. The order of occurrence of the nine "events" is superfluous as the events are indistinguishable (because the trials are independent and, for the moment, seasonality is not a concern). Therefore, the input data matrix of Fig. 1(b) can be condensed. Guided by this observation, we let the rank be an independent variable and construct a $64 \times 64$ rank-order square matrix $P$ as shown in Fig. 1(c), where each entry is the "occupation number" or the number of occurrences for that particular rank and year. The total population of the $P$ matrix is $365 \times 64$. Note that $P$ is integer valued and invariant with respect to the temperature offset, and the total population of each row and column is 365 . More generally, for $P$, the range is $\left[0, n_{t}\right]$, where $n_{t}$ is the number of trials (here, days).

Note that the entries of $P$ are not evenly distributed among the quadrants defined by the crosshairs in Fig. 1(c). Whereas the combined population of upper-left and lowerright quadrants is 14083 , that of lower-right and upper-left quadrants is 9277. The expected population, given a stationary climate, is $(365 \times 64) / 2=11680$. This nonstationarity of approximately $20.6 \%$ is of overwhelming statistical significance, and we use this message in the data to work towards an objective, assumption-free definition of a warming signal. The extreme case of a pure warming trend with no variability results in a $P$ that is a multiple of the identity matrix, with a prefactor $n_{t}$. By contrast, consider an ensemble of stationary climate realizations. For a given time series of 64 years, any entry is equally likely to be the hottest (record-breaking), and shuffling these entries does not change the statistics because of independence [13]. Then, in the limit, ensemble-averaged populations of all ranks of a given row of $P$ (fixed time) should be equal, and the matrix $P$ should approach perfect uniformity (all matrix elements equal, $P=$ const).

To gain further insight into the meaning of the $P$ signal, consider an early and late year, namely, 1954 (order 4) and 2011 (order 61), displayed as a histogram versus rank in Fig. 1(d). Observe that, for a steady climate, rank occupation numbers, approximated as independent trials (akin to classical particles), obey Poisson statistics: $p(n)=$ $\left(\mu^{n} / n !\right) e^{-\mu}$, with $\mu=365 / 64=5.70$ being the average population per rank and $\sigma=(365 / 64)^{1 / 2}=2.39$ the standard deviation. Hence, we expect approximately $6 \pm 2$, as the green curve (labeled stationary) indicates. This result is not so for the red (diamond) and blue (circle) curves. Note a near perfect reflection symmetry between these curves. This symmetry is another manifestation of warming. The 1954 and the 2011 population maxima occur at ranks 3 and 63 , respectively. Hence, the statistically essential information for these years is stored in intermediate ranks (see Fig. 1 caption for further numerical illustration). On the other hand, high occupation of mid-rank, say, rank 32, although significant, does not convey as much information about a warming trend as the high occupation of extreme, or near extreme, ranks. 

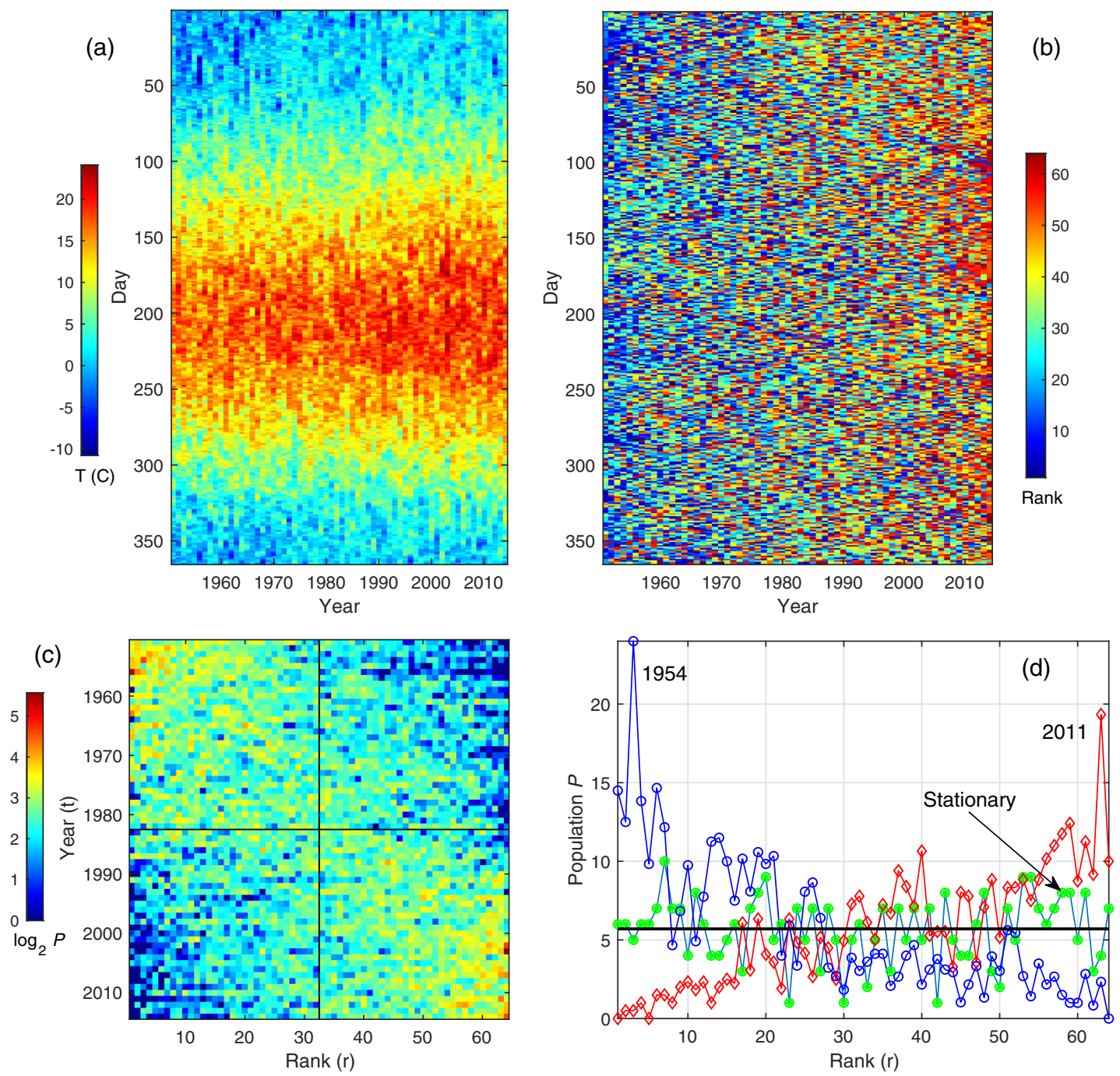

FIG. 1. Data processing, illustrated on weather station SZ000009480 (GHCN) Lugano, Switzerland. (a) Daily high-temperature values displayed as a day (row) and year (column) matrix. (b) Same data but with only the daily rank recorded in rows. (c) The $64 \times 64$ rank-year square matrix $P$, where each entry is the color-coded number of occurrences of that particular rank in that year ("occupation number"). The combined population of upper-left and lower-right quadrants (defined by the crosshairs) is 14083 , whereas the combined population of the lower-left and upper-right quadrants is 9277, with the expected population for stationary climate $(365 \times 64) / 2=11680$. This quadrupolar asymmetry constitutes a warming signal. (d) 1954 and 2011 population vs rank. The near reflection symmetry between the red (diamond) and blue (circle) curves is evident and compatible with warming, with $75 \%$ of the 1954 population in the bottom half of ranks and 75\% in the top half for 2011. The 1954 and 2011 maxima are at ranks 3 and 63, respectively. Considering the mean of $\mu=365 / 64=5.70$ and the Poisson pdf (valid for iid climate, see text), 24 occurrences of rank 3 in the year 1954 are exceedingly unlikely for a stationary climate $\left(7 \times 10^{-7}\right)$. On the other hand, the number of record highs in 2011 was 10 , which is plausible (3\%) for a stationary climate [occurring once also in the green (asterisk) curve, which is one realization of a stationary climate]. Hence, most of the essential information here is contained in the intermediate ranks. The argument is stronger yet for autocorrelated data.

Based on the above discussion, the notion of a warming signature emerges, characterized by the overpopulation of the lowest ranks in early years, i.e., red values in upper-left and lower-right corners, with the blue values predominantly in the other two corners. But why limit one's attention to only the symmetric partition of $P$ into four quadrants? To that end, consider the general partitioning into (unequal) quadrants defined by the off-center crosshairs in Fig. 2(a) and focus on the excess of records over the expected mean in quadrants 2 and 4, and the corresponding deficit in quadrants 1 and 3. For each quadrant pair, we take the ratio of actual to expected populations and then form the difference of these two ratios. This difference vanishes (on average) for a steady climate. For the data of Fig. 1, the value of this difference at the centered crosshairs $(32,32)$ is $14083 / 11680-9277 / 11680=0.4115$, while a peak 

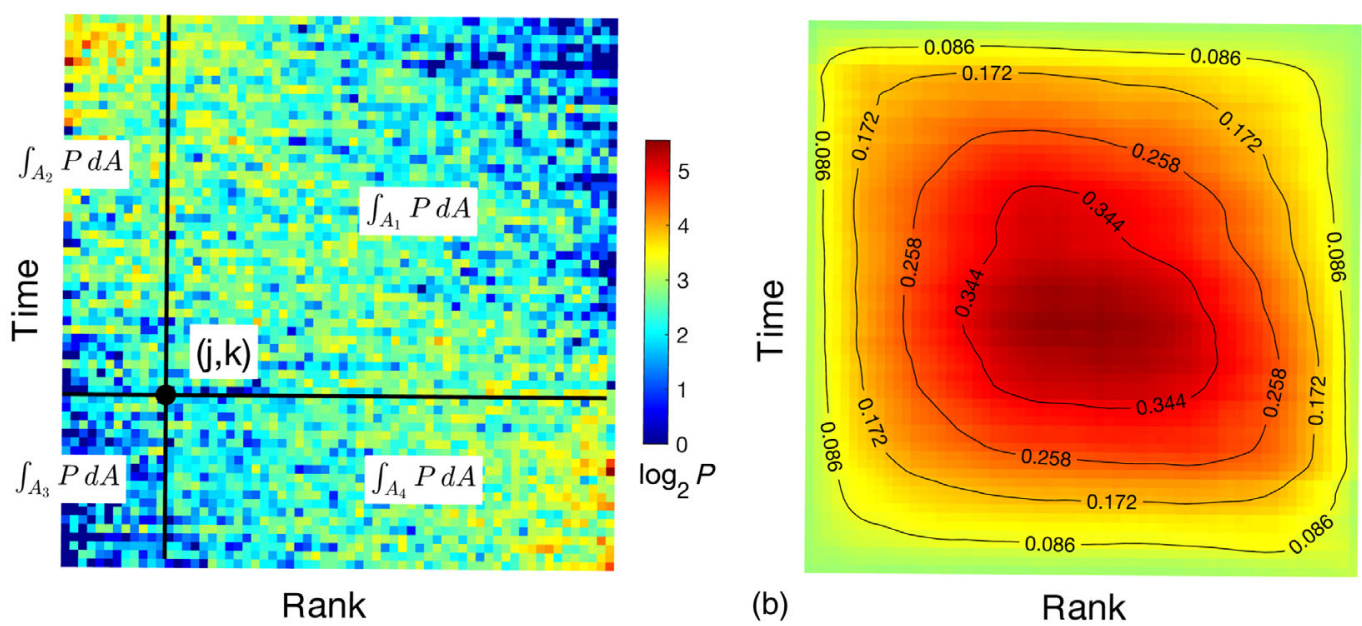

(a)

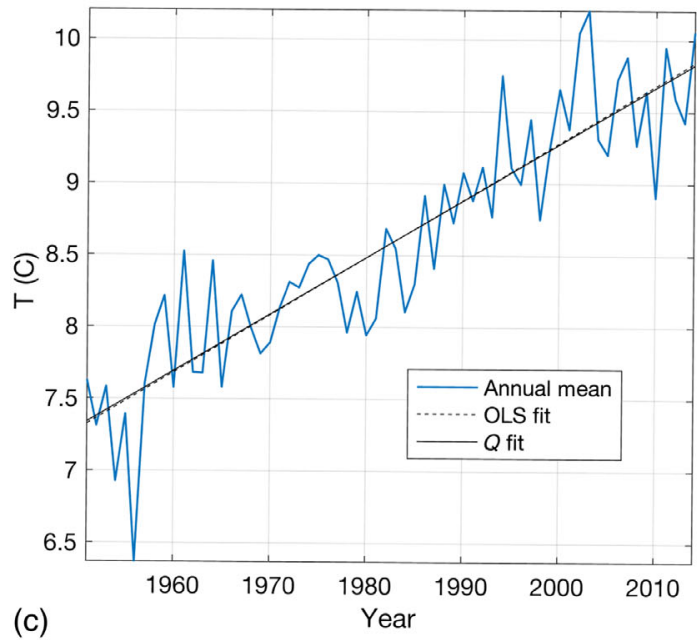

(b)

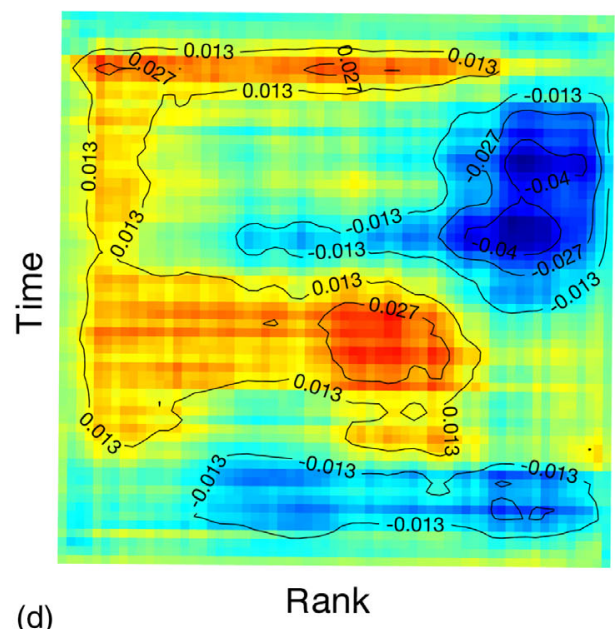

FIG. 2. $\quad P$ to $Q$ transformation and resulting trend, for SZ000009480 (GHCN), Lugano, Switzerland. (a) Crosshairs centered at the $(j, k)$ grid element; partitioning of $P$, used to compute the $(j, k)$ element of $Q$. (b) $Q$ computed via Eq. (1), revealing a prominent warming pattern. (c) The linear trend, obtained by annulling the matrix element average $\langle Q\rangle\left(2.4875^{\circ} \mathrm{C}\right)$, which is nearly identical to the standard LS fit $\left(2.5165^{\circ} \mathrm{C}\right)$. (d) The residual $Q$ computed from the raw-temperature record after linear detrending [color scale expanded from that for panel (b) to preserve detail]. Note the large-scale residual pattern, which is an order of magnitude smaller than the original $Q$. All plots of $Q$ throughout the paper employ dark blue and dark red to denote bounds of $[-\max |Q|$, max $|Q|]$, respectively. Henceforth, pale green will thus indicate zero in these plots.

value of 0.4283 occurs at $(35,34)$. When this partitioning is repeated with the crosshairs traversing the entire grid, a new matrix is generated, denoted as $Q$, e.g., $Q_{32,32}=0.4115$. To ensure the existence of the four quadrants, given that $P$ is $N \times N$, the difference of ratios is computed at $(N-1) \times$ $(N-1)$ grid points [14]. The mathematical implementation for the above construction of $Q$ is given by

$$
\begin{aligned}
Q_{j, k}= & \frac{n_{T}}{n_{t}}\left[\frac{\sum_{m=1}^{j} \sum_{n=1}^{k} P_{m, n}+\sum_{m=j+1}^{n_{T}} \sum_{n=k+1}^{n_{T}} P_{m, n}}{j k+\left(n_{T}-j\right)\left(n_{T}-k\right)}\right. \\
& \left.-\frac{\sum_{m=j+1}^{n_{T}} \sum_{n=1}^{k} P_{m, n}+\sum_{m=1}^{j} \sum_{n=k+1}^{n_{T}} P_{m, n}}{j\left(n_{T}-k\right)+\left(n_{T}-j\right) k}\right],
\end{aligned}
$$

where $n_{T}$ is the number of years. This result defines the discrete $Q$ transform of $P$. Note that $-2 \leq Q_{j, k} \leq 2$; i.e., $Q_{j, k} / 2$ is the excess or deficit percentage for the $(j, k)$ partition of $P$. If $Q$ and $P$ are rearranged as vectors, Eq. (1) can be viewed as $\mathbf{q}=M \mathbf{p}$, where the matrix $M$, augmented with the row and column sum constraints for $P$, is well conditioned and admits a stable inversion for $P$ given $Q$. Hence, $Q$ preserves, while reordering, the trend information stored in $P$ from the original temperature record.

For the weather station of Fig. 1, the corresponding $Q$ is shown in Fig. 2(b). The complete trend information is stored in the set of partitions of $P$ and hence in the elements of $Q$. As illustrated above, positive elements of $Q$ arise from partitions with a warming bias. Thus, for a stationary 
climate, one anticipates no sign preference for elements of $Q$. This motivates us to consider $\langle Q\rangle$, the mean value of all matrix elements, defined as

$$
\langle Q\rangle \equiv \frac{1}{N^{2}} \sum_{i=1}^{N} \sum_{j=1}^{N} Q_{i, j} .
$$

The angular brackets, from now on, denote the average over all matrix elements throughout this paper (as opposed to an ensemble average); thus, $\langle Q\rangle$ is a scalar, and it vanishes, on average, for a stationary random process.

Natural variability induces fluctuations in $\langle Q\rangle$ about zero. Once that probability distribution is characterized, one has a quantitative basis to decide whether a trend is actually present, as discussed below. Thus, we propose to quantify a trend by the linear function (temperature vs time) whose slope is determined by annulling the mean value of $Q$. In other words, a single adjustable parameter, the slope, is chosen to annul the average matrix element of $Q$. To do this, a candidate linear function of $T(t)$ is subtracted from the original time series (input data), row-by-row ranks are recomputed, $P$ is repopulated with revised values, the $Q$ transform is applied, and its mean $\langle Q\rangle$ is computed. The scalar $\langle Q\rangle$ is a monotone function of the trial slope and always has a single zero crossing.

To illustrate, we return to the data in Fig. 2. Remarkably, $Q$ is positive definite, that is, positive for each and every partition of $P$ (each matrix element of $Q$ ). Thus, the warming signature is exceptionally strong. Moreover, as Fig. 2(c) confirms, not only does annulling $\langle Q\rangle$ in the original record determine a unique linear trend, that trend is nearly indistinguishable from the LS fit. Similar close agreement between LS and $Q$ linear trends is found in most cases. Nonetheless, while LS and $Q$ fits of temperature trend commonly agree to $0.05^{\circ} \mathrm{C}$ over periods of 50 years or more, a few larger discrepancies arise. These arise in cases with large seasonal variation in variance, which we explore shortly. A systematic cause of smaller discrepancies is that LS regression of the annual mean does not distinguish between a few large excursions in daily low temperature vs numerous small excursions, whereas $Q$ is affected principally by the latter. Lastly, autocorrelation, common in temperature time series, can differentially affect the two.

The partitioning of temperature data in a $365 \times 64$ matrix may seem a necessary condition for linear regression with $Q$, but this is not so. Dropping one calendar day to obtain $364 \times 64$ points affords a wide number of factorizations. The set $n_{T}=[26,28,32,52,56,64,91,104,112,128]$ serves to make the point. Before detrending, $\langle Q\rangle$ values for this set consist of seven approximately equal low values, one intermediate, and two high. The last pair are the original $n_{T}=64$, and subharmonic, $n_{T}=32$, which averages two years of temperatures at a time. The superharmonic, $n_{T}=128$, averages every six months; hence, the signal has both a long-term trend and a period-two seasonality. Its initial $\langle Q\rangle$ is intermediate. The remaining seven, incommensurate with seasonality, all have a very irregular mean signal, though it is still marked by the same long-term trend. Each factorized form was detrended with exactly the same slope. All of them simultaneously have $\langle Q\rangle$ reduced to noise level (or, translated back to temperatures, differences averaging about $\pm 0.01{ }^{\circ} \mathrm{C}$ ). So the choice of binning causes no meaningful disagreement about the trend required to annul $\langle Q\rangle$ on the assumption of a linear long-term signal.

Note that the algorithm of obtaining the linear trend with $Q$ is objective in the sense that a robot can be programmed to detrend the temperature data by simply annulling $\langle Q\rangle$. A skeptical reader might wonder about extracting a dimensional quantitative trend in degrees/decade from the dimensionless rank input only. In fact, it is signal and noise that together conspire to give $Q$ the quantitative information needed because ranks are scrambled by the noise indiscriminately while the signal affects them systematically. The key relation here is a proportionality constant that relates a dimensional change in slope to the dimensionless change induced in $\langle Q\rangle$ for a specified noise field. Unlike $Q$ itself, that constant depends upon the exact distribution. We revisit this point at the end of Sec. XI, where an error estimate for the slope is derived.

As we see, the rank-order transform $Q$ reveals the entire form of a signal and not just the linear trend; i.e., there is information in the residual $Q$ shown in Fig. 2(d). One does not generally expect $Q$ and least squares fits to agree at all orders, particularly as ordinary least squares fits are influenced by outliers, while $Q$ is not (see the treatment of heavy tails in Sec. X, where empirically weighted least squares fits can work only up to a point, while $Q$ performs well without the need for such measures). Note also that a monotone deformation of temperature data (e.g., a logarithmic one) affects the least squares fit but not $Q$.

To illustrate some remarkable properties of the $Q$ transform, we consider a highly idealized synthetic data set because the true answer is known (hence, $Q$ and LS errors can be assessed quantitatively) and because the idealization makes the cause of the difference in comparative performance transparent. Motivated by the data for Bethel Airport, AK, where $Q$ and (unweighted) LS trends for 1951-2014 differ by $0.72^{\circ} \mathrm{C}$, we consider the daily low temperature on Planet X, where the climate is so equable for the first half of the year as to have no variability in temperature but solely a trend of $1{ }^{\circ} \mathrm{C}$ over 64 years. In contrast, during the second half of the year, the same trend is overlain with large variance. Figure $3(\mathrm{~b})$ shows the corresponding $Q$ and LS fits: $1.0001{ }^{\circ} \mathrm{C}$ and $1.69^{\circ} \mathrm{C}$, respectively. Clearly, the LS fit is thrown off by the abrupt noise. Figure 3(c) depicts the $P$ matrix (note the log scale), revealing the reason for the divergent estimates. The exact data for the first half of the year result in a perfectly 

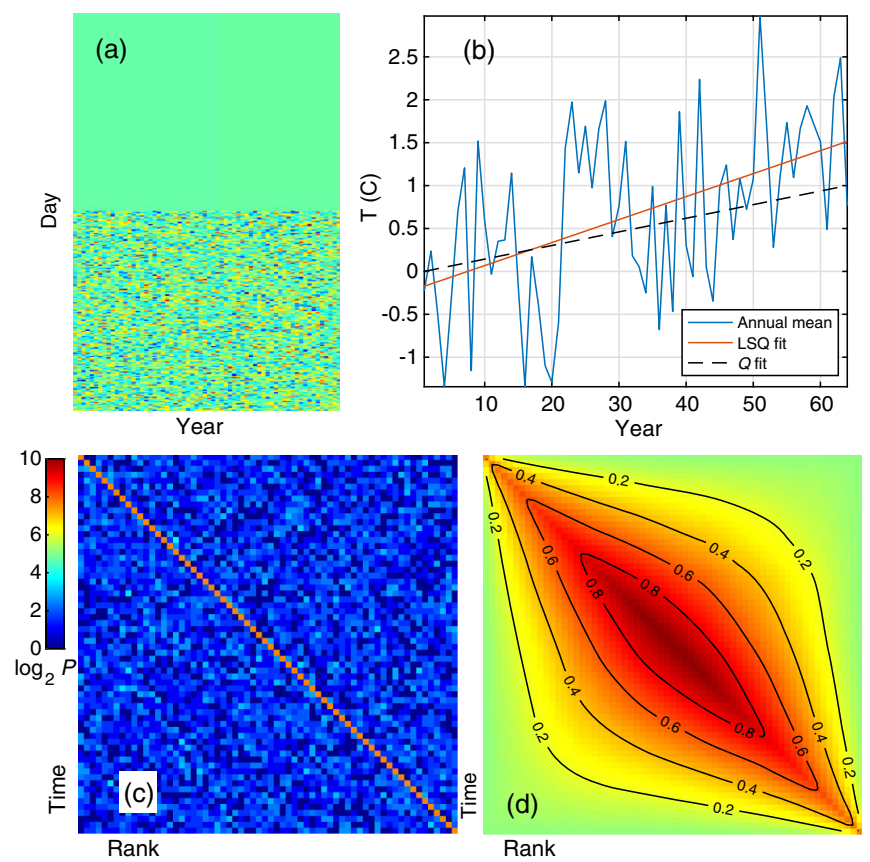

FIG. 3. An example of superior $Q$ performance: Synthetic data for daily low temperature on Planet $X$ (see text). (a) The data matrix. For the first half of the year, the temperatures are noisefree; only the trend of $1{ }^{\circ} \mathrm{C}$ over 64 years is present. During the second half of the year, large fluctuations are superimposed on the same trend. (b) A comparison of $Q$ and LS fits for this imaginary station. The $Q$ fit gives $1.0001^{\circ} \mathrm{C}$. The LS fit is thrown off by the noise, giving $1.69^{\circ} \mathrm{C}$ with a confidence interval of [1.06, 2.31]. (c) The $P$ (log scale) matrix, which shows the reason for the disparity. The exact data for the first half of the year result in a diagonal population of entries, while the second half of the year consists of randomly sprinkled entries. (d) $Q$ is hardly perturbed by noise; each partition sees a positive excess dominated by the diagonal.

diagonal population of entries, while the second half of the year consists of nearly randomly distributed entries. Figure 3(d) shows that the resulting $Q$ is resistant to noise; each partition sees a positive excess strongly dominated by the diagonal, while the random entries largely average out. Hence, detrending this $Q$ [see Eq. (9) for an exact expression in the limit of zero noise] effectively yields an exact result. In real data, all cases of large discrepancies in trend estimates between $Q$ and LS occur in locations that experience large excursions in seasonal variance. Conversely, $Q$ and LS linear trends for stations with minimal variance excursions commonly agree within the previously indicated $0.05^{\circ} \mathrm{C}$ per 50 years.

\section{SIMPLE ANALYTIC APPROXIMATIONS FOR $Q$}

Towards gaining an intuitive sense for $Q$, we introduce here a continuous version of $Q_{j, k}$, denoted as $q(x, y)$, and similarly for $P$. For simplicity, the domain of each is taken as $[-1,1] \times[-1,1]$. Then,

$$
\begin{aligned}
q(x, y)= & \frac{1}{2-2 x y}\left[\int_{-1}^{x} d x^{\prime} \int_{y}^{1} d y^{\prime} p\left(x^{\prime}, y^{\prime}\right)\right. \\
& \left.+\int_{x}^{1} d x^{\prime} \int_{-1}^{y} d y^{\prime} p\left(x^{\prime}, y^{\prime}\right)\right] \\
& -\frac{1}{2+2 x y}\left[\int_{-1}^{x} d x^{\prime} \int_{-1}^{y} d y^{\prime} p\left(x^{\prime}, y^{\prime}\right)\right. \\
& \left.+\int_{x}^{1} d x^{\prime} \int_{y}^{1} d y^{\prime} p\left(x^{\prime}, y^{\prime}\right)\right]
\end{aligned}
$$

and we require $p(x, y)$ to satisfy the homogeneous constraints

$$
\int_{-1}^{1} d x p(x, y)=0, \quad \int_{-1}^{1} d y p(x, y)=0 .
$$

Making use of the latter constraints, Eq. (3) can be simplified to

$$
\begin{aligned}
q(x, y)= & \frac{1}{1-x^{2} y^{2}}\left[\int_{-1}^{x} d x^{\prime} \int_{y}^{1} d y^{\prime} p\left(x^{\prime}, y^{\prime}\right)\right. \\
& \left.+\int_{x}^{1} d x^{\prime} \int_{-1}^{y} d y^{\prime} p\left(x^{\prime}, y^{\prime}\right)\right] .
\end{aligned}
$$

The inversion yields

$$
p(x, y)=\frac{1}{2} \frac{\partial^{2}}{\partial x \partial y}\left[\left(1-x^{2} y^{2}\right) q(x, y)\right]
$$

Alternatively, we can write Eq. (3) in the form of a twodimensional convolution as

$$
\begin{aligned}
q(x, y)= & \frac{1}{1-x^{2} y^{2}} \int_{-1}^{1} \int_{-1}^{1}\left[H\left(x-x^{\prime}\right) H\left(y^{\prime}-y\right)\right. \\
& \left.+H\left(x^{\prime}-x\right) H\left(y-y^{\prime}\right)\right] p\left(x^{\prime}, y^{\prime}\right) d x^{\prime} d y^{\prime}
\end{aligned}
$$

where $H$ denotes the Heaviside function.

The simplest possible algebraic form that satisfies Eq. (4) is $p(x, y)=-x y$, and we choose the sign to reflect an excess in second and fourth quadrants and a deficit in the first and third, that is, a warming signal. From these assumptions, we get 


$$
q(x, y)=\frac{1}{2} \frac{\left(1-x^{2}\right)\left(1-y^{2}\right)}{1-x^{2} y^{2}},
$$

with a mean value of $\pi^{2} / 8-1 \approx 0.2337$ and root-meansquare value of $\sqrt{1-3 \pi^{2} / 32} \approx 0.2733$. While the issue of normalization has been bypassed, this simple ansatz for $p(x, y)$ is an excellent means to anticipate the form of a ubiquitous pattern in $Q$, both for real data at numerous sites with warming, and the dominant mode of $Q$ in a PCA decomposition, even for realistic correlated temperature fluctuations in a stationary climate, typically accounting for $25 \%$ of the variance in $Q$. While Eq. (8) reflects the form of $Q$ for a wide range of SNR, the limiting form for zero noise is a diagonal matrix for $P$. Translated to the continuous form, this results in

$$
\begin{aligned}
q(x, y)= & \frac{2}{x^{2} y^{2}-1}[1+x y-(x-1) H(x-1) \\
& -(x+1) H(x+1)+2(x-y) H(x-y) \\
& +(y+1) H(-y-1)-(1-y) H(1-y)],
\end{aligned}
$$

whose diamond-shaped contours are those seen in Fig. 3. In this special case, the formula above, if sampled on the unit interval at a spacing of $\Delta x=2 / n_{T}$ with endpoints excluded, is identical to the discrete result for $n_{T}$, regardless of the value of $n_{t}$. Appendix A examines breaks in a series, based on this continuous approximation.

\section{METRICS OF $Q$, THEIR STATISTICAL DISTRIBUTIONS AND ASYMPTOTICS}

Reduction to a $P$ matrix is the basis for the $Q$ transform, and the exact general result for the equilibrium form of $P$ for a given signal in the presence of uncorrelated noise can be obtained. This result is essential for deriving error bounds. However, because of numerical complexity for realistic arguments and the need for development of its asymptotic expansion, we defer that discussion to Appendix B.

Here, we extend our approach, which began by consideration of $\langle Q\rangle$ in Sec. II. We aim to characterize the standard deviation for $\langle Q\rangle$ for iid noise. To this end, we find an asymptotic expansion that clarifies parametric dependencies. Deeper meaning of such benchmarking emerges in the next section.

In Sec. II, we proposed that a linear trend can be determined by setting the average matrix element $\langle Q\rangle=0$. Such a trend is a combination of a long-term signal plus some contribution from natural variability. Given but a single realization, one cannot disentangle these two. However, knowing the distribution of $\langle Q\rangle$, one can set bounds on the contribution from natural variability to within any desired confidence level. For iid noise, the quantity $\langle Q\rangle$ follows a normal distribution, and the standard deviation of $\langle Q\rangle$ can be characterized in general terms. Considering the disparate influence of $n_{t}$ and $n_{T}$ on that result, one expects the dependence on the former to be the same as that for a sum of $n_{t}$ normal variables, namely, $n_{t}^{-1 / 2}$. It is plausible that an asymptotic expansion of $\sigma_{\langle Q\rangle}$ in $n_{T}$ has the same leadingorder dependence, succeeded by an ordered progression of higher-order corrections. Numerical experiments at varying $n_{T}$ and $n_{t}$ with $6 \times 10^{5}$ realizations each time yield the following approximation in such a form:

$\sigma_{\langle Q\rangle} \sim \frac{0.7131}{n_{t}{ }^{1 / 2}}\left[\frac{1}{n_{T}{ }^{1 / 2}}-\frac{0.2299}{n_{T}}+\frac{3.3026}{n_{T}^{3 / 2}}+\mathcal{O}\left(\frac{1}{n_{T}{ }^{2}}\right)\right]$.

(The coefficients above are sensitive to errors in computed estimates of $\sigma_{\langle Q\rangle}$.) As $Q$ is an ordinal method, asymptotic results such as Eq. (10), and also Eq. (12) below, are distribution independent for white noise. The form above can be motivated by comparison to the derivation for a related expansion (see Appendix B). A sample run with 5000 trials using iid normal random variables $n_{t}=365$ and $n_{T}=50$ give $\sigma_{\langle Q\rangle}=0.005456$ compared to the expected result from Eq. (10) of 0.0054556. Normalizing values of $\langle Q\rangle$ with the sample standard deviation yield a distribution that passes the Kolmogorov-Smirnov test for normality at the 5\% significance level with an asymptotic $p$ value of 0.035 .

Beyond linear trends, $Q$ may reveal a general nonlinear signal, and a suitable second benchmark is then the rootmean-square (rms) value of $Q$ whose distribution must be characterized. The rms average $\langle Q\rangle$ is given by

$$
Q_{\mathrm{rms}} \equiv \sqrt{\left\langle Q^{2}\right\rangle}=\frac{1}{\left(n_{T}-1\right)}\left[\sum_{j=1}^{n_{T}-1} \sum_{k=1}^{n_{T}-1} Q_{j, k}^{2}\right]^{1 / 2} .
$$

The pair of mean and rms values of $Q$ have the great advantage that they are readily computed, especially the first, for which there is a fast explicit algorithm given in Appendix B. [There is also a fast $\mathcal{O}\left(n_{T}^{2}\right)$ algorithm for $Q$ itself given $P$.]

The quantity $Q_{\mathrm{rms}}$ is observed to obey a generalized $\chi$ distribution, and it collapses to a single curve as a function of the normalized variable $\tilde{Q}_{\mathrm{rms}} \equiv Q_{\mathrm{rms}} /\left\langle Q_{\mathrm{rms}}\right\rangle$, where a similar asymptotic expansion holds, namely,

$$
\left\langle Q_{\mathrm{rms}}\right\rangle \sim \frac{1.3725}{n_{t}{ }^{1 / 2}}\left[\frac{1}{n_{T}{ }^{1 / 2}}+\frac{0.0293}{n_{T}}+\frac{1.3577}{n_{T}{ }^{3 / 2}}+\mathcal{O}\left(\frac{1}{n_{T}{ }^{2}}\right)\right] .
$$

An empirical expression for the cumulative distribution function (cdf) with uniform error $<0.004$ can be written in terms of the incomplete gamma function [15] as 


$$
\begin{aligned}
\operatorname{cdf}\left(\tilde{Q}_{\mathrm{rms}}\right) \approx 1 & -\frac{\Gamma(9.6070,13.6038 \ln x+9.9521)}{\Gamma(9.6070)} \\
& \times(x>0.481) .
\end{aligned}
$$

One must qualify the use of results like Eqs. (10) and (12) when the ambient noise is other than iid (white) noise. One common factor is autocorrelation. For example, it is a matter of common experience that weather has a persistence, typically 3 to 4 days. With the temperature data running vertically in the data matrix of Fig. 1(a), one has a resulting correlation between successive rows in that data matrix. For correlated identically distributed variables arranged in this fashion, it remains true that $\langle Q\rangle$ follows a normal distribution, but the coefficients in Eq. (10) depend on the specific autocorrelation.

For $Q_{\text {rms }}$, not only do the coefficients change but the generalized $\chi$ distribution itself is altered, as seen in Fig. 4 where two examples make our point. The more conventional case is provided by convolving a Gaussian white noise sequence with a Gaussian filter of the form $\exp \left(-0.0346\left(n-n^{\prime}\right)^{2}\right)$. As in Fig. 1(a), the data are stacked vertically in the input matrix to $P$; hence,

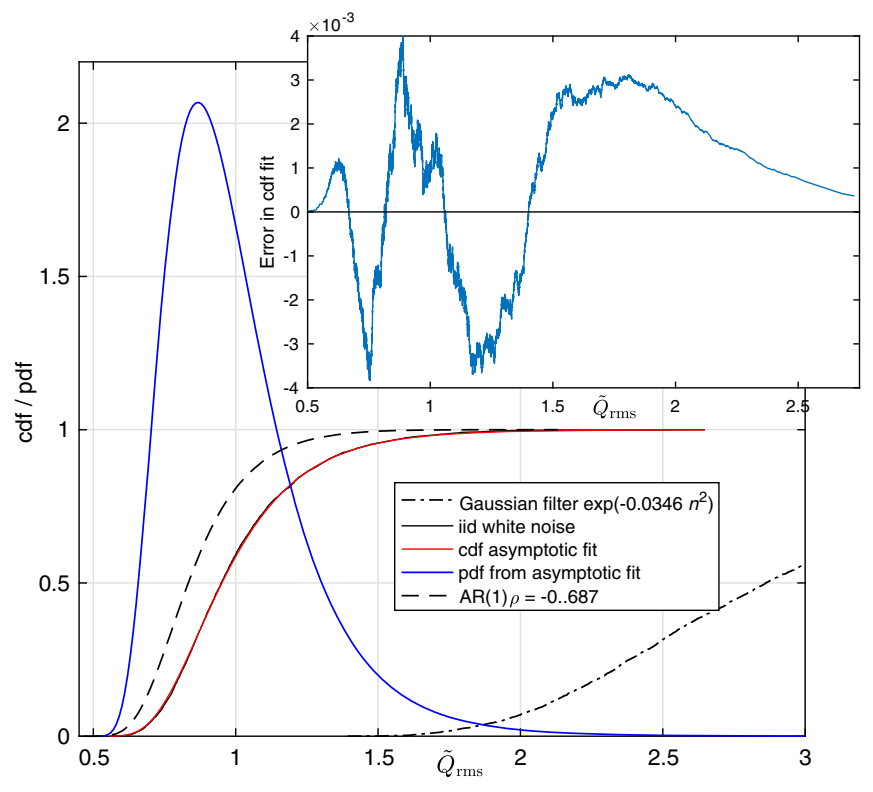

FIG. 4. Distribution of $Q_{\mathrm{rms}}$ : A baseline cdf of $Q_{\mathrm{rms}}$ for iid noise and the asymptotic fit given at Eq. (13) (and corresponding pdf). The fit error is shown in the inset figure. Two comparison distributions show the effect of autocorrelation. The first distribution (dashed line), with a narrower scale than the standard (solid line), is generated by an $\operatorname{AR}(1)$ model with $\rho=-0.68716$, which has a strong negative correlation at one time lag. The second distribution, which is broader (dash-dotted line), is generated by iid noise convolved with a Gaussian filter. The latter two coincide closely with the standard under a linear remapping of the abscissa. From Eq. (12), this result indicates that the effect of autocorrelation amounts to a change in the effective $n_{t}$. Asymmetry of the distribution is clear in the pdf based on the asymptotic fit. successive rows are correlated. The resulting distribution of $Q_{\text {rms }}$ (dash-dotted line) is observed to be broader. A second example, with a narrower distribution, is an $\operatorname{AR}(1)$ model with $\rho=-0.68716$, whose autocorrelation function has a pronounced dip of -0.7 at one time lag. The cdf for the standard reference $\tilde{Q}_{\text {rms }}$ (solid black), along with its asymptotic fit (13), lies between the other two. The difference between empirical and asymptotic results for iid noise is shown in the inset figure, as is the probability density function (pdf) that follows from the asymptotic form (13).

All three cdf curves are scaled by the same iid noise value for $\left\langle Q_{\mathrm{rms}}\right\rangle$. For these two correlated examples, a linear remapping of the form $\tilde{Q}_{\mathrm{rms}} \rightarrow \alpha \tilde{Q}_{\mathrm{rms}}+\beta$ gives a curve fairly close to the original iid distribution. The parameters that achieve this are $(\alpha=0.2574, \beta=0.0046)$ for the Gaussian filter, and $(\alpha=1.3138, \beta=-0.1423)$ for the AR(1) model. The first of these parameters, a shrinking of scale, can be thought of as a decrease in the effective number of independent samples $n_{t}$ [16]. That the second comparison distribution is narrower is attributable to the negative correlation, which disrupts, rather than reinforces, the tendency for sample variability. We draw upon this dynamic to great effect in Sec. VII, where we consider chaotic series generated by the logistic map, also generally characterized by negative correlation.

\section{SAMPLE VARIABILITY PROJECTED ON THE RANK-ORDER $Q$-PLANE, CHARACTERIZING STATIONARY RANDOM PROCESSES}

The data shown in Fig. 2(c) exhibit an unmistakable linear trend. Yet, at least in principle, natural variability of a truly stationary climate could create such a trend. While strict stationarity is a theoretical property of a random process, finite samples (even large ones) never appear purely random and exactly stationary. Finite samples exhibit transient trends, and the likelihood of such trends depends on the specific stationary process. But, while spurious trends in sample mean and variance can be a hindrance for deterministic signal detection, one can turn this around and use these same calculated trend likelihoods to characterize (or "fingerprint") specific stationary stochastic processes.

As we demonstrate below, the "lifting" of a onedimensional time series to the two-dimensional space of rank order via the $Q$ transform enables an application of group theory, delivering a universal characterization of transient trends for arbitrary stationary stochastic processes and sample sizes. In particular, we pay special attention to two models: the Ornstein-Uhlenbeck process and the logistic map, the latter of which is explored further, from the perspective of deterministic chaos, in Sec. VII.

Towards the complete characterization of transient trends, we begin with the iid (stationary, $\delta$-correlated, or white) noise, which is the featureless "standard candle" of stochastic processes. Because the $Q$ transform is ordinal, 

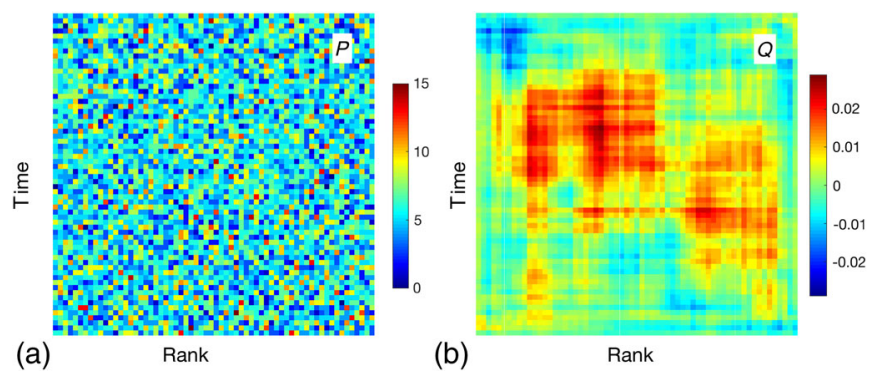

FIG. 5. Contrast between $Q$ and $P$ representations. (a) The fine speckle from a single realization of $P$ from the stationary iid Monte Carlo simulation, as described in the text. (b) The corresponding spatial coherence in $Q$ (for any noise pdf). The average population per pixel on the left is $(365 / 64) \approx 5.7$. Whereas $P$ is finely speckled, $Q$ exhibits a spatial structure, which, in this realization, is associated with a warming trend. Structures become more pronounced at the ensemble level (see Fig. 6).

there is no need to limit our development to a Gaussian distribution; all white noise distributions are equivalent. The featureless spectrum of white noise suggests the absence of features in any representation. Indeed, this featureless quality is true at the level of raw input data and remains true for the $P$ matrix [e.g., see Fig. 5(a)], devoid of apparent structure, appearing as salt-and-pepper noise. In fact, as all ranks have equal rights, ensembleaveraged $P$ tends to the perfect uniformity (constant $P$ ) for, not just iid, but more generally to all independent stationary processes because of the reshuffling argument (see Sec. II).

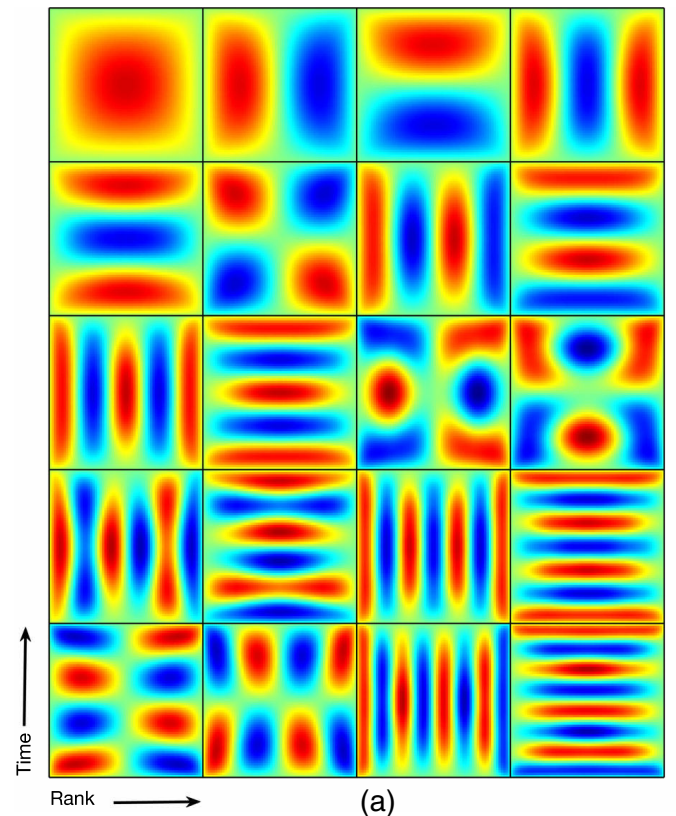

This limit also holds for correlated (and hence, shufflingbreaking) stationary processes, aside from slight effects at the corners (see Appendix B 1).

In contrast, the ensemble average of the $Q$-transformed (distribution-invariant) white noise in the rank-order plane (hereafter, dubbed $\pi$ noise [34]) is not uniform, and even at a single realization level, it deviates greatly from the saltand-pepper noise, as illustrated by the patchiness (structure) in Fig. 5(b). We take advantage of such structure and decompose it in terms of dominant modes (planforms), linking these planforms to the types of transient patterns in time (see Fig. 6).

\section{A. Group-based algorithm for the standard "etalon"}

The desired correspondence between the planforms of $Q$ and specific features in the generating time series emerges from an examination of symmetries and associated groups. Group character is central in the rank-order plane; e.g., timereversal symmetry means the ensemble average of $P$ is invariant under a left-right flip. Just as any $1 \mathrm{D}$ function $f(x)$ can be written as the sum of even and odd terms, $1 / 2[f(x)+f(-x)]+1 / 2[f(x)-f(-x)]$, an arbitrary function in $n$ dimensions has a distinguished orthogonal group decomposition in $n !+2^{n}-1$ terms (two terms for $n=1$ ). For $n=2$, the five-term expansion assumes the form

$$
q(x, y)=q^{\left(D_{4}\right)}+q^{\left(D_{2}\right)}+q_{x}^{\left(C_{1}\right)}+q_{y}^{\left(C_{1}\right)}+q^{\left(R_{2}\right)},
$$

where

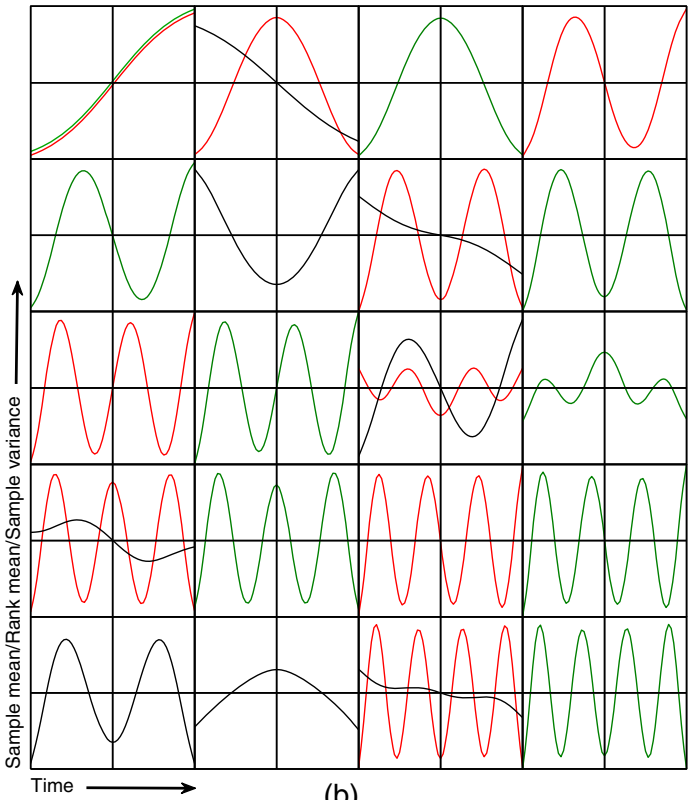

(b)

FIG. 6. Universal modal decomposition of iid noise in the $Q$ representation: A complete characterization of finite sample nonstationarity. (a) Combined $Q$ matrix PCA modes $\psi_{k}$ for $k=1, \ldots, 20$ from all five symmetry group projections, ordered (left to right) by decreasing singular value. (b) Leading-order sample variability pattern of the corresponding time series: Data mean $\delta \mu_{k}$ (green lines), data variance $\delta \sigma_{k}$ (black lines), and rank mean $\delta r_{k}$ (red lines). 


$$
\begin{aligned}
q^{\left(D_{4}\right)}= & {[q(x, y)+q(-x, y)+q(x,-y)+q(-x,-y)} \\
& +q(y, x)+q(-y, x)+q(y,-x)+q(-y,-x)] / 8, \\
q^{\left(D_{2}\right)}= & {[q(x, y)+q(-x, y)+q(x,-y)+q(-x,-y)} \\
& -q(y, x)-q(-y, x)-q(y,-x)-q(-y,-x)] / 8, \\
q_{x}^{\left(C_{1}\right)}= & {[q(x, y)+q(-x, y)-q(x,-y)-q(-x,-y)] / 4, } \\
q_{y}^{\left(C_{1}\right)}= & {[q(x, y)-q(-x, y)+q(x,-y)-q(-x,-y)] / 4, } \\
q^{\left(R_{2}\right)}= & {[q(x, y)-q(-x, y)-q(x,-y)+q(-x,-y)] / 4 . }
\end{aligned}
$$

Here, $D_{n}$ denotes the dihedral group, $C_{n}$ the reflection group, and $R_{n}$ the rotation group, with the third and fourth components on the right in Eq. (14) representing reflections about the $x$ and $y$ axes, respectively [17]. The applications of this expansion appear to be manifold, including an exploration of wallpaper groups as in Refs. [18,19]. The first term is the only one in the decomposition with (in general) a nonzero mean value when integrated over the domain; all others vanish identically by antisymmetry.

The expansion (14) can be applied in discrete form to the square matrix $Q$ for each realization [20], yielding five ensembles, one per group. Each of these ensembles is then characterized by principal component analysis (PCA). This expansion is driven by data (hence, Lorenz's term "empirical orthogonal functions" [21]), rather than preselected, as in a generalized harmonic analysis of noise. PCA is designed to decorrelate the signal by projecting the data onto orthogonal axes. Here, it decomposes $\pi$-noise variability in the $Q$ group representation with modes in order of decreasing contribution to variance $\left(\sigma_{Q}^{2}\right.$, a quadratic metric) of each ensemble.

For numerical implementation, PCA is evaluated by singular value decomposition (MATLAB routine svd). We used an ensemble of $10^{5}$ realizations populated by iid normal random variables of zero mean and unit variance, though the ordinal results depend on neither choice, even from row to row. For $\delta$-correlated noise, the lowest modes from the PCA decompositions of the resulting $Q$ group ensembles rapidly approach their limiting forms as a function of $n_{T}$, with the highest retained mode $\psi_{20}$ determining the needed grid resolution. We aim for wellresolved structure, not just meeting the Nyquist limit. As a test of this, spline interpolation of $\psi_{1}$ for $n_{T}=65$ onto the coarser mesh of $\psi_{1}$ for $n_{T}=49$ gives a relative standard error for the mismatch of $3 \times 10^{-3}$. The singular values (scaled by $n_{T}^{-1 / 2}$ ) exhibit a similar relative error. The choice of $n_{T}=65$ will thus suffice for most applications, so one need not repeatedly compute this etalon for different $n_{T}$ but can rather rely on interpolation.

When searching for signal in noise, $Q$ approaches a finite limiting form as $n_{t}^{-1 / 2}$. Here, there is no signal and hence no structure that $Q$ attains with increasing $n_{t}$. Remarkably then, and quite in contrast to, e.g., the temperature data for
Lugano, the PCA results for iid noise with the minimum possible choice of $n_{t}=2$ are indistinguishable from those for $n_{t}=2048$. The reduction to $n_{t}=2$ saves CPU time for both generating the random realizations and their initial processing to obtain $P$.

For each symmetry group, the PCA modes have an ordered set of singular values. The collected set of all group PCA modes is then resorted by singular value, with the corresponding symmetry group noted for each. In this merged set, one encounters repeated mode pairs of symmetry $\left(\psi_{D_{4}}, \psi_{D_{2}}\right)$ and $\left(\psi_{R_{2}}, \psi_{R_{2}}\right)$. In both cases, transient nonstationarity is more compactly represented by forming sum and difference modes, i.e., $\left(\psi_{D_{4}} \pm \psi_{D_{2}}\right) / \sqrt{2}$, and similarly for the other pair. There are also unpaired modes of all three symmetries, particularly at higher order. But, of the first 20 modes, only two such exceptions occur: the first and sixth modes, which we turn to shortly.

\section{B. Results: A universal characterization of transients for $\pi$ noise}

Figure 6(a) shows a set of $x-y$ oriented planforms, indexed as $(j, k)$ denoting a total of $j$ extrema in the $x$ direction and $k$ in $y$. The case of $j=k$ corresponds to the above two unpaired modes $j=\{1,2\}$, while for $j \neq k$, we have pairs in the form of a matrix and its companion transpose. This then constitutes our "etalon" against which stochastic processes are to be compared [22].

The $\pi$-noise variability falls into three main categories: nonstationarity of the sample mean, $\delta \mu_{k}$; nonstationarity of sample variance, $\delta \sigma_{k}^{2}$; and departure from $\delta$ correlation, described by the autocorrelation function (ACF) for stationary random processes [23]. For reasons of symmetry in the rank-order plane, we also consider nonstationarity of (sample) mean rank, $\delta r_{k}$. These curves are obtained by conditional sampling in a long Monte Carlo run. Each realization with a mode projection for $\psi_{k}$ exceeding the $2 \sigma$ level is captured. The means of the realizations thus isolated, mode by mode, are plotted in the matching tableaux of Fig. 6(b). These curves (time series) follow the group selection rules indicated in Table I [24].

\section{Tutorial on Fig. 6: Case studies for modes 1 and 6}

Although PCA modes for $P$ are of little use, each $Q$ mode $\psi_{k}$ can be inverted to discover its antecedent $P$ [25]. In Figs. 7(c) and 7(d), we show the $P$ precursors for two modes, $\psi_{1}$ and $\psi_{6}$, reproduced here from Fig. 6(a). These examples will demonstrate how separation of transient mean and transient variance arises from "lifting" to the rank-order plane. (Other similar separations are also seen in Fig. 6, e.g., modes $\psi_{17}$ and $\psi_{18}$, associated solely with variance.)

The first mode $\psi_{1}$ is associated with an approximately linear trend in data, $\delta \mu_{1}(x)$ [26]. How can one see this intuitively? Here, $P_{1}$ proves essential. Imagine a realization 
TABLE I. Column 1: Planform patterns for the modes $\psi_{k}$ in Fig. 6. Column 2: Their symmetry group-dihedral group $D_{n}$, reflection group $C_{n}$, and rotation group $R_{n}$. Column 3: Symmetry group of the companion precursor $P$ field. Column 4: Associated fluctuation fields, which vanish identically.

\begin{tabular}{lccc}
\hline \hline Planform & $Q$ sym & $\delta P$ sym & Null projection \\
\hline$(2 j+1,2 j+1)$ & $D_{4}$ & $R_{2}$ & $\delta \sigma_{k}^{(\pi)}(x)=0$ \\
$(2 j+1,2 k+1) j \neq k$ & $D_{2}$ & $R_{2}$ & $\delta \sigma_{k}^{(\pi)}(x)=0$ \\
$(2 j+1,2 k)$ & $C_{1}^{(y)}$ & $C_{1}^{(x)}$ & $\delta \mu_{k}(x)=0$ \\
$(2 k, 2 j+1)$ & $C_{1}^{(x)}$ & $C_{1}^{(y)}$ & $\delta r_{k}(x)=\delta \sigma_{k}(x)=0$ \\
$(2 j, 2 k)$ & $R_{2}$ & $D_{4} \pm D_{2}$ & $\delta r_{k}(x)=\delta \mu_{k}(x)=0$ \\
\hline \hline
\end{tabular}

for which Fig. 7(e) is, by chance, the mean trend. Record lows (and generally lower ranks) are more likely to occur at early times and, conversely, record highs at later times. Such an excess of record lows in the upper (early time) left (lower rank) corner of $P$ is shown in red, and similarly for the lower-right corner, paralleling the construction that led to Fig. 2. Thus, a linear trend of the mean yields $P_{1}$, odd in
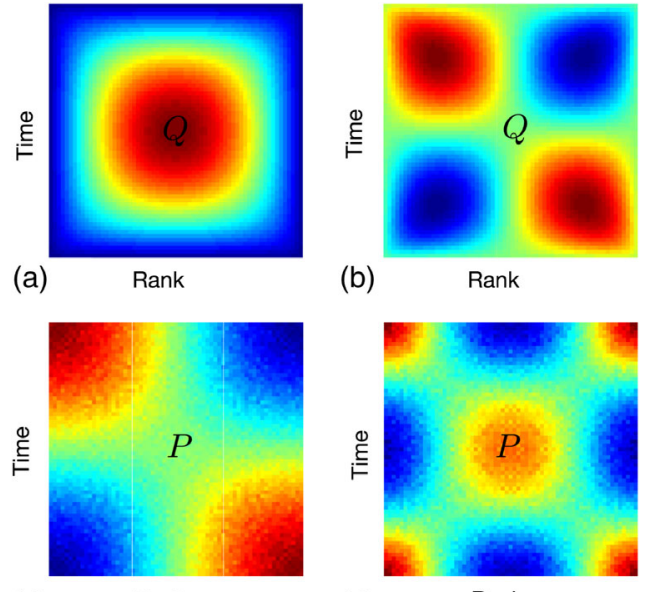

(c) Rank

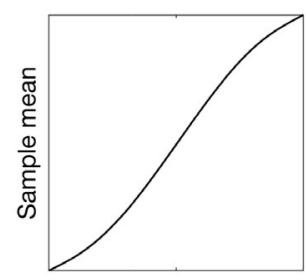

(e) Time (arb. units) (b)

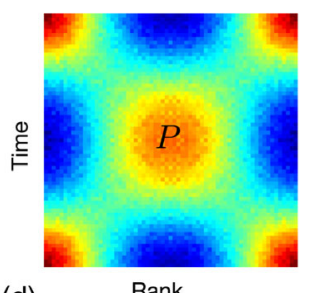

(d)

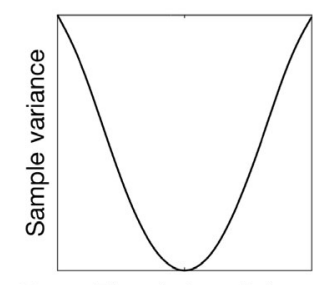

(f) Time (arb. units)

FIG. 7. Examples of modes 1 and 6: (a) $\psi_{1}$ with $D_{4}$ symmetry; (b) $\psi_{6}$ with $R_{2}$ symmetry [both from Fig. 6(a)]; (c) inverting $\psi_{1}$ for $P_{1}$ from Eq. (1), which gives a result with $R_{2}$ symmetry; (d) same procedure for $P_{6}$ with $D_{4}$ symmetry. Similarly, panel (e) shows the conditionally averaged sample mean of the data for $\psi_{1}$ (averaged over $n_{t}$ trials) and resembles the Lugano temperature time series of Sec. II. In panel (f), the vaselike profile in sample variance, also from conditional sampling, represents overpopulation of the four corners of $P_{6}$. [Both panels (e) and (f) are from Fig. 6(b).]

both its dimensions and corresponding to an even/even $Q$ [consistent with the mixed derivative in Eq. (6)]. Not only linear trends but also antisymmetric ones lead, in general, to $R_{2}$ symmetry of $P$ and $D_{4}$ symmetry of $Q$.

The second mode $\psi_{6}$ is paired with a roughly quadratic profile in variance. Again, by appealing to $P_{6}$, we can understand this relation by considering a realization with sample mean variance as in Fig. 7(f). Now both record highs and lows are more likely to occur at early and late times, thereby producing the red corner pattern of Fig. 7(d). Furthermore, the overpopulation of middle ranks at intermediate times also marks the center of $P$ red. Then, because of the row and column sum constraints, necessarily all four middle edges must be under-populated (blue). A similar derivative argument applies for parity, and a general statement is that symmetric trends in variance lead to $D_{4}$ symmetry of $P$ and $R_{2}$ symmetry of $Q$.

Returning now to Fig. 6(b), note the consecutive identical pairs of mean rank (red) and mean data (green), that is, $\delta \mu_{2}(x)=\delta r_{3}(x)$ for $\psi_{2}$ and $\psi_{3}$, respectively, and so on. The first exceptions are $\delta r_{11}$ and $\delta \mu_{12}$, which are inverted versions of each other [27]. Similarly, the only member of the odd/odd planform category here is $\psi_{1}$, but the notation in Table I anticipates the presence of a higher planform $(3,3)$ also of $D_{4}$ symmetry. Mode 21 from the merged PCA expansion is that planform. The four leading PCA modes of this merged set account for nearly half the total variance, while the asymptotic decay rate is about $n^{-\ln 2}$ [28], in contrast to the "whitish" one of about $n^{-\varepsilon}$ for raw input or $P$.

Transient trends in (sample) variance are plotted in black. Note how modes of $R_{2}$ symmetry $(6,17,18)$ are associated with spurious trends in sample variance alone, just as modes of $D_{4}$ and $D_{2}$ symmetry are linked to an odd order trend of only the sample mean. It is in the $C_{1}$ pairs that odd order variance and even order mean are linked.

The notation $\delta(r, \sigma)_{k}(x)$ reminds one that these modes are zero-mean fluctuations. But ensemble means from conditional sampling are not zero mean. Rather, the conditionally sampled modes for rank all have mean $\left(1+n_{T}\right) / 2$, and similarly, the modes for variance have a mean equal to that for a sum of $n_{t}$ values of a random variable from the particular distribution used, here unity. The negative values in the plots for Fig. 6(b) are then relative to these means. Both rank and variance themselves remain positive definite. For graphical purposes, only a single rescaling was applied to all curves in Fig. 6(b), so their relative magnitudes can be compared directly.

While results based on rank, as for any results from $Q$, are distribution independent, transient dimensional fluctuations in mean and variance refer back to the raw data space, and these necessarily reintroduce a dependence on the particular distribution in question. The issue is a constant of proportionality between, say, a given gradient in dimensional variables and the induced change in the 
dimensionless measure $\langle Q\rangle$. We treat this for the specific case of Gaussian noise later in Sec. XI, where we derive an explicit error estimate for $Q$-based linear regression. The theoretical framework for making that link is given in Appendix B.

Note that $\langle Q\rangle$ automatically annihilates all modes except those of groups $D_{4}$ and $D_{2}$. The latter group occurs in pairs. Each such mode pair $\left(\psi_{k}, \psi_{k+1}\right)$ can be rotated back to the original basis by $\left(\psi_{k} \mp \psi_{k+1}\right) / \sqrt{2}$. Only the recovered mode of $D_{4}$ symmetry then contributes to $\langle Q\rangle$. The second -in which trends in rank and mean are anticorrelated vanishes identically upon integration.

Anticorrelation is forbidden at lowest order; the only mode present already is of group $D_{4}$. The linear trend in rank must hence match the trend in the data regardless of the loss of magnitude information. This idea is not obvious. One can try to construct a companion $Q$ mode, necessarily of group $D_{2}$, with rank and data linearly anticorrelated, e.g., $x^{2}-y^{2}$ in continuous form but inversion of any such form yields a $P$ of singular support, that is, a set of measure zero for projections from the space of ranked white-noise realizations. The problem is that one needs a form for $Q$, which vanishes on the boundaries but at the same time satisfies (in the continuous version)

$$
\frac{d}{d y} \int_{-1}^{1} q(x, y) d x \sim y \text { and } \frac{d}{d x} \int_{-1}^{1} q(x, y) d y \sim-x,
$$

and this is evidently not possible.

We can now give a precise statement of the meaning of annulling $\langle Q\rangle$ : The initial data yield a nonzero $\langle Q\rangle$ from the sum of projections on $D_{4}$ modes only (subject to the second rotation noted above) [29]. Adding a linear trend to the data modifies the contributions, principally from mode 1 . The coefficient of that linear term is adjusted until the total sum from all $D_{4}$ terms vanishes. As explained in the discussion of Fig. 7, this procedure is unaffected by nonstationary variance. The invariance of $Q$-derived trends of the mean with respect to variance thus holds unconditionally.

This point is the crucial difference between least squares and $Q$. Least squares fits are strongly affected by nonstationary variance, as shown by our earlier toy model of Fig. 3. One then has to resort to empirically determined weights to try to minimize this influence. For heavy-tailed noise, however, such weights prove ultimately ineffective, as we later document in Sec. X. No such empirical machinery is needed for $Q$.

Note that if the goal is merely to obtain a trend by annulling $\langle Q\rangle$, then any functional form with a nonzero antisymmetric component will also project on the $D_{4}$ modes and hence determine a unique amplitude for that function. In other words, annulling $\langle Q\rangle$ does not grant any special status to a linear trend. Rather, that choice resides in the application, and the onus is on the user to choose.
A second moment of interest is $\langle Y \circ Q\rangle$ (where $\circ$ represents the Hadamard product). This moment only selects for the modes in group $C_{1}$ with parity +- , which are raised to $D_{2}$ and parity ++ , and thus contribute in integral. This is the natural companion measure to detect even signals of nonstationary mean while $\langle Q\rangle$ detects odd.

Note the generality of these results: The $Q$ response to an actual signal of low SNR results from combining the components in Fig. 6(a) weighted by the expansion coefficients for that signal when expressed in terms of the complete set $\left\{\delta \mu_{k}\right\}$ in Fig. 6(b). Hence, whether considering the transient sample mean of a stationary process or the real mean of a nonstationary one, $Q$ detects them the same way. The key distinction is that, for the case of $\pi$ noise, the standard deviation for each of these modes is universal and fixed, and their means vanish; for a signal, the amplitudes are arbitrary and unknown in advance.

\section{FINGERPRINTING STOCHASTIC PROCESSES}

The group PCA decomposition yields the $\pi$-noise standard deviation for each of the first 20 modes, thus defining benchmarks. Stationary processes other than white noise will deviate in one or more of these measures, just as observed earlier in Sec. IV, with the influence of autocorrelation on the distribution of $Q_{\mathrm{rms}}$. Although group PCA components represent apparent nonstationarity, spontaneously arising in a finite sample of a random process, each standard deviation for the parent distribution of individual mode coefficients has an asymptotic expansion of the same general form as Eqs. (10) and (12). Hence, the suite of ratios of such quantities (a "fingerprint") approaches a well-defined limit as $n_{T} \rightarrow \infty$.

The four processes illustrated in Fig. 8 are as follows: the Ornstein-Uhlenbeck $(\mathrm{O}-\mathrm{U})$ process with relaxation $\tau=1$ and $c=2$ (as in Ref. [30]), the autoregressive process AR (1) with $\varphi=-0.68761[31,32]$, a model for patchiness consisting of white noise with the standard deviation for each successive group of 13 samples chosen from a uniform distribution on the interval $[0,1]$, and a chaotic series generated by the logistic map with $r=3.731$.

This group-theoretic signature, consisting of the standard deviation for each mode normalized by the $\pi$-noise values, is one way to detect and/or classify a specific stationary stochastic process. The signature is a function of $n_{T}$ (but not $n_{t}$ ) just as, in the correlation theory of random processes, the ACF is a function of the number of time lags, $n_{\tau}$. But the fingerprint furnishes information beyond that available from the ACF. Distinct stochastic processes with nearly identical ACFs are shown in Fig. 8: (1) the $\delta$ correlated (like $\pi$ noise) patchy process whose fingerprint oscillates about the $\pi$-noise standard; (2) the AR(1) model and the logistic map with distinct fingerprints.

The largest departures from $\pi$ noise occur for the O-U process, with a long correlation, in contrast to the $\delta$ correlated patchy process. This fingerprint of O-U can 


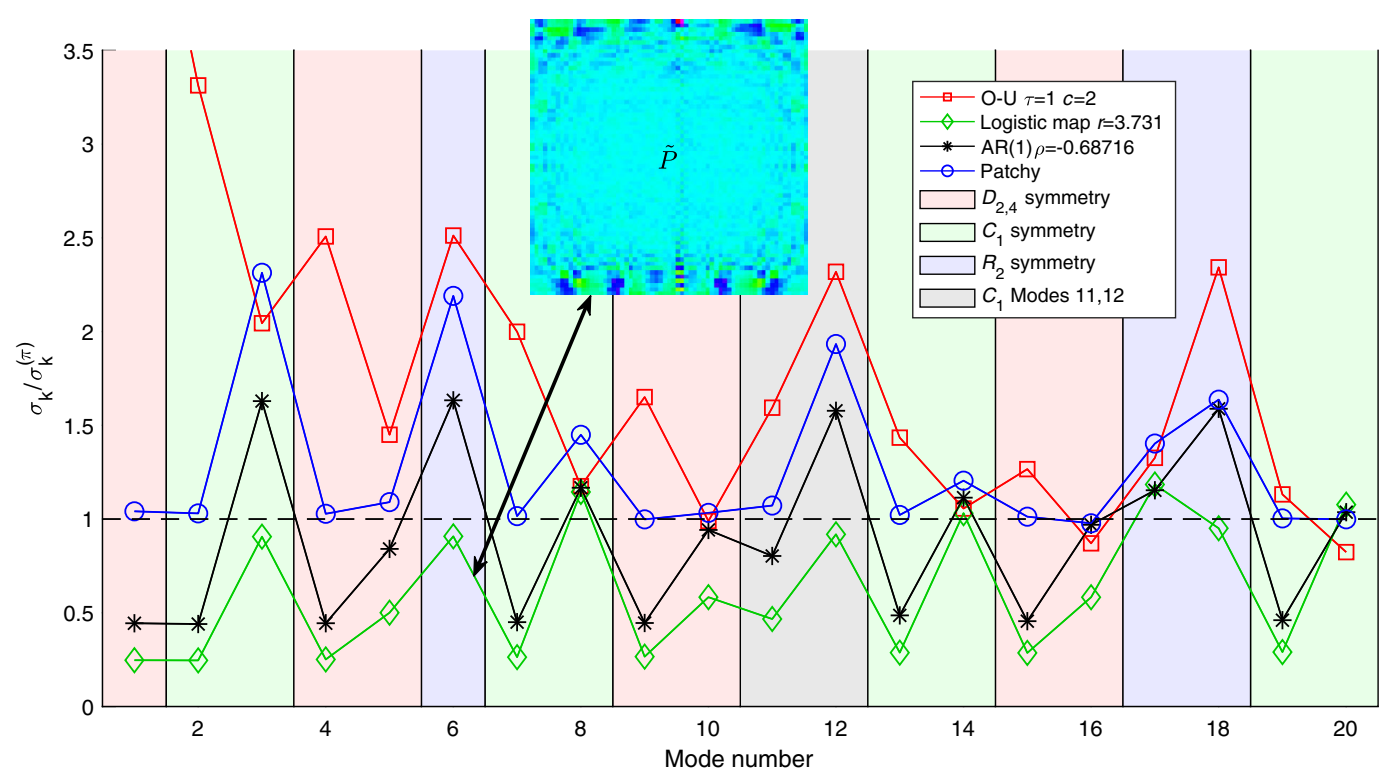

FIG. 8. Stationary stochastic processes and deterministic chaos characterized by patterns of rank sample variability. The $y$ axis gives the standard deviation $\sigma$ of the modal coefficients normalized by the $\pi$-noise values. Three models are stationary stochastic processes: (1) Ornstein-Uhlenbeck, (2) "patchy" $\delta$-correlated, and (3) first-order autogressive [AR(1)]. The fourth is the chaotic logistic map (examined further in Sec. VII). For the parameters noted in the legend, the AR(1) and logistic models have essentially equal ACFs but distinct fingerprints. Both dip below the $\pi$ noise because of the negative correlation at small lags, reducing the likelihood of a spurious trend. The inset shows $\tilde{P}$ (the ensemble mean $P$ without its $D_{4}$ component) for the logistic map. For all independent stationary stochastic processes, $\tilde{P}=0$, but for all deterministic processes, like here, $\tilde{P} \neq 0$. The patchy process illustrates that $\delta$-correlated processes can still have nontrivial fingerprints. The most telling feature of the O-U process is its greatly increased probability of spurious linear trends relative to $\pi$ noise as indicated by its off-scale value for mode 1 of 4.97 .

be compared to the approach to stochastic signal detection in Ref. [30], but with the further development in Ref. [33], generalized there from a parametric to a nonparametric version based on higher moments of noisy data. Our method is also nonparametric, but it only deals with rank and hence serves as a complementary approach to Ref. [33].

Fingerprints of stochastic processes should be compared at the same $n_{T}$ (or $n_{T} \Delta t$ in the continuous case). As $n_{T}$ attains a value several times the longest expected correlation, the fingerprint attains its asymptotic limit. For three of the four processes in Fig. 8, $n_{T}=65$ is well into that regime. However, the continuous $\mathrm{O}-\mathrm{U}$ process has a much longer correlation time, and, for a step size of $\Delta t=0.01$, one would need $n_{T}$ of order $10^{3}$ to reach that limit. Its fingerprint at $n_{T}=65$, strongly dominated by the (offscale) peak mode 1 , is nonetheless a perfectly fair point of comparison with any other stochastic process at the same $n_{T}$ [34].

\section{A. Generality of results}

The suppression of apparent linear trends (mode 1) by both the logistic map and AR(1) in Fig. 8 evidently reflects an inhibiting effect of the negative correlation at one time lag in the ACF. But the hallmark of true, rather than apparent sample, nonstationarity is the presence of structure in $P$, as for any deterministic signal, buried in noise or not. This case is in contrast to the constant (uniform) ensemble-averaged matrix $P$ that is obtained for any stationary random process (but see Appendix B 1 for a small caveat, which explains the removal of the $D_{4}$ component of $P$ as in Fig. 7, hence the $\tilde{P}$ in Fig. 8). Chaotic systems are deterministic, and even the logistic map at $r=4$, commonly thought to be random, has a structured $\tilde{P}$. All chaotic systems exhibit intricate, and unique, ensemble-averaged patterns for $\tilde{P}$. One of the discoveries of this paper is that ranking within randomness differs inherently from ranking in chaos [as well as more orderly deterministic systems) as reflected in rank portraits (analogous to phase portraits), e.g., $\tilde{P}$ in Fig. 8].

As a possible application, consider a time series of velocities measured in high Reynolds number, statistically stationary, turbulent flow. It is a standard assumption that the power spectrum of such a flow obeys the Kolmogorov $k^{-5 / 3}$ scaling at intermediate wave numbers. Typically, a suitable log-log plot is used to test this and even to deduce small $\ll \mathcal{O}(1)$ corrections to the power scaling, caused by fine-scale intermittency. Given the inevitable measurement noise, how clearly is this scaling distinguishable from, say, $k^{-6 / 3}$ scaling? The latter is mimicked by the Lorentzian power spectrum whose ACF is exponential, i.e., a firstorder Markov process. Here, one could run the $Q$ transform, to fingerprint the time series without prejudice, at the 
"machine learning" stage, before committing to a stochastic process model.

\section{AN ILLUSTRATION FROM DETERMINISTIC CHAOS: THE LOGISTIC MAP}

In many physics applications, "noise" means fluctuations in the measurement or observation (e.g., Ref. [35]), while "signal" suggests deterministic components. Chaos produced, for example, by a nonlinear dynamical system is neither. Following a suggestion by an anonymous reviewer, we digress to test the $Q$ transform on deterministic chaos generated by the famous logistic map:

$$
x_{n+1}=r x_{n}\left(1-x_{n}\right) .
$$

Another approach to the detection of chaos derives from a measure of complexity originally devised by Bandt and Pompe [36] for detection of speech, and as a robust substitute for the Lyapunov exponent. This recipe was later used in Ref. [37] to develop a "complexity-entropy causality plane" that can clearly distinguish chaotic from stochastic systems.

While earlier we relied upon metrics such as $\langle Q\rangle$ rising above a threshold value dictated by the desired confidence level as the means for signal detection, with deterministic chaos, the tables are turned. A chaotic trajectory is, of course, in a loose sense, "noisy," but the implication for the pdf of $\langle Q\rangle$ is that it is not noisy enough; it fails to span the gamut of values that would be seen with, say, $\pi$ noise. A general signature of this is that the standard deviation falls below the asymptotic estimate in Eq. (10). When this occurs, we conclude that deterministic chaos is present in the time series, either alone or in concert with stochastic noise.

The bifurcation sequence through which a chaotic map is reached at $r_{\infty}=3.569945672$ is discussed in, e.g., Ref. [38]. We take a time series from Eq. (15) with $128 \times$ 64 entries and reconstitute it in matrix form, again with the entries stacked vertically. The pdf for $\langle Q\rangle$ as a function of $r$ is instructive, as seen in Figs. 9 and 10. For $r=3.58$, immediately above the onset at $r_{\infty}$, the pdf is extremely narrow and multipeaked. These peaks are vestiges of the principal bifurcation branches at lower $r$. But by $r=3.8$, all such evidence is absent; the pdf is normal with a standard error of $\sigma=1.45 \times 10^{-4}$. As anticipated, these chaotic data have a systematically narrower range of $\langle Q\rangle$ values than found for random noise, which, based on Eq. (10), would have $\sigma_{\langle Q\rangle}=8.059 \times 10^{-3}$ (the scale factor for the $x$ axis here). But, with increasing $r$, the width grows and, at the
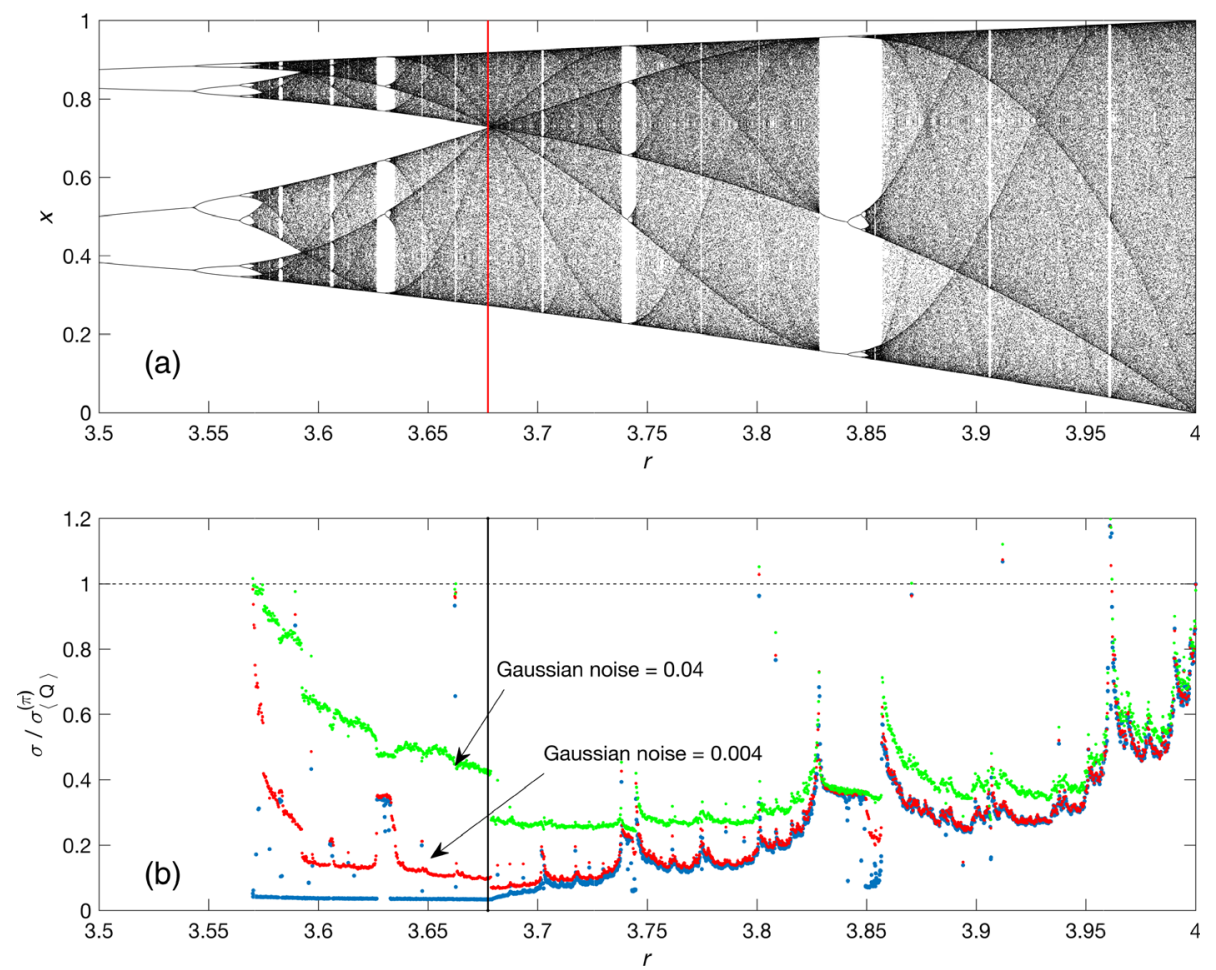

FIG. 9. $Q$ distinguishes chaos from noise: Distribution of $\sigma / \sigma_{\langle Q\rangle}$ for the logistic map. (a) The logistic map vs $r$. (b) Estimate for the standard deviation for $\langle Q\rangle$, normalized by its value for $\pi$ noise as given in Eq. (10). Each of the lacunae in the map in panel (a) has its counterpart as an interrupted trace in the curves below. Note that the dividing line at $r_{c}=3.6875$ marks a boundary between spiked and normal pdfs. (See Fig. 10.) The lowest trace is that for pure deterministic chaos; the two above show its modification in the presence of additive Gaussian noise with $\sigma=0.004$ (as in Ref. [36]) and $\sigma=0.04$. Note the separatrix at $r_{c}$ : To the left, the spiked pdfs are more quickly altered by a given stochastic noise level, while the normal pdfs on the right respond only slightly. 


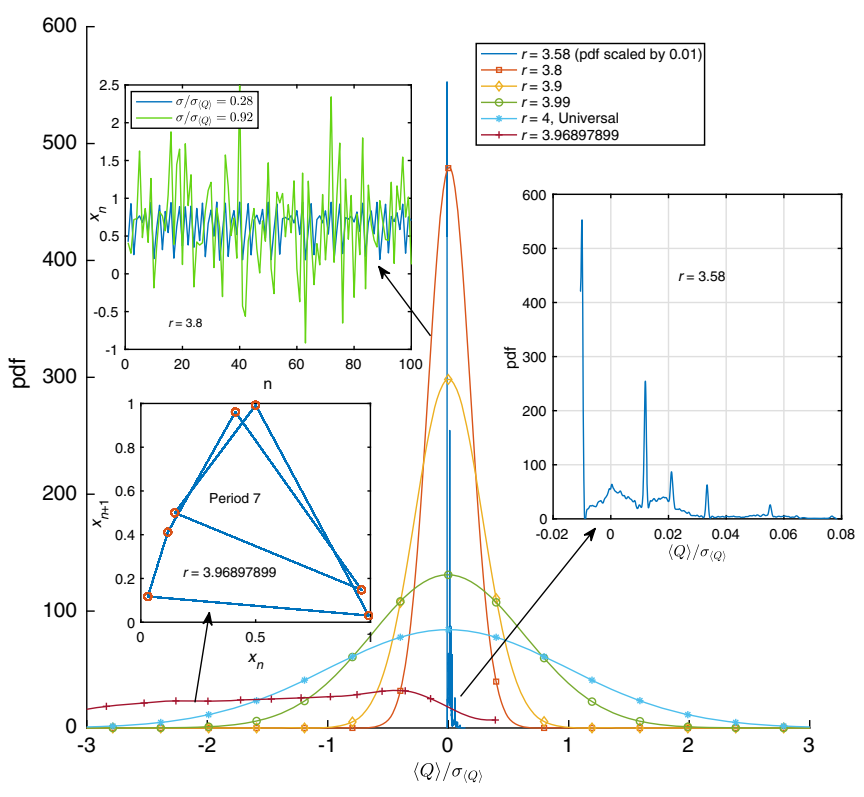

FIG. 10. $Q$ distinguishes chaos from noise: pdf of $\langle Q\rangle$ vs $r$. A representative spiked pdf when $r<r_{c}$ is shown for $r=3.58$. Note the spiky character. Immediately for $r>r_{c}$, this gives way to normal distributions, such as those plotted here for $r=[3.8,3.9,3.99,4]$. The distribution for $r=4$ has a standard deviation exactly matching the prediction from (10), indicating that the output of the logistic map then exactly matches iid noise statistics. However, the path to this is punctuated by spikes in the $\sigma$ plot at, e.g., $r=3.96897899$, where $\sigma / \sigma_{\langle Q\rangle}=16$ (well off scale in this truncated plot). This case constitutes a "normal signal" in the form of a period-seven orbit (lower left). In the upper left are two traces: pure deterministic chaos, and the same with added Gaussian noise with $\sigma=0.5$. Despite intense noise, the chaos imprint is readily discernible.

end point of $r=4$, the pdf for $\langle Q\rangle$ coincides exactly with the earlier described "universal distribution" for noise. This general picture needs to be qualified as suggested by filamentary structure in Fig. 9.

The initial transition from a spiked pdf to a normal distribution occurs at $r_{c} \approx 3.6875$, as marked by the vertical line in Fig. 9(a), where upper and lower branch families first meet. As noted by a referee, there is a parallel feature that pairs with this transition in the pdfs for $\langle Q\rangle$; below $r_{c}$, the pdf for $x_{n}$ itself is singular; above $r_{c}$, the pdf, still punctuated with singularities, has full support. Yet another representation of this stochastic "phase transition" is the fingerprint of Sec. VI, which for the logistic map has a discontinuity at $r=r_{c}$.

However, there are discrete departures again from the normal pdf, e.g., those associated with the gaps centered at $r=3.74$ and $r=3.84$. There is a large isolated spike at $r=3.96897899$ with the indicated anomalously broad pdf, stemming from an orbit of period seven. It achieves a peak of $\sigma / \sigma_{\langle Q\rangle}=16$; i.e., this represents normal signal detection by $Q$. Similar features punctuate the curve elsewhere. Each feature in Fig. 9(b) can be linked with associated structure in the logistic map above, but the general pattern, again, consists of Gaussian pdfs of increasing standard deviation to the right.

Figures 9 and 10 depict the standard deviation for the distribution of $\langle Q\rangle$, with Eq. (10) used as the benchmark for $\pi$ noise. By continuity, near the terminus at $r=4$ and bracketing the spike at $r=3.96897899$ must lie two adjacent values of $r$ at which $\sigma / \sigma_{\langle Q\rangle}=1$. These are not the loci of $\pi$ noise, however, as the coincidence with the value from Eq. (10) is a necessary but not sufficient condition. A practical sufficiency condition is that the pdf itself, when $\sigma / \sigma_{\langle Q\rangle}=1$, also passes the KolmogorovSmirnov test for normality. This condition is true only at $r=4$, not elsewhere.

The red and green traces in Fig. 9 show the displacement of the curves due to the addition of Gaussian noise of the indicated magnitude. Note the increasingly sharp discontinuity at $r_{c}$, with Gaussian (or smooth) pdfs only minimally disrupted by noise while the singular ones exhibit heightened sensitivity. Furthermore, the inset at the top left in Fig. 10 shows two traces: the logistic map for $r=3.8$ and the same output with added Gaussian noise of $\sigma=0.5$. Even for intense noise- a decrease in SNR of $42 \mathrm{~dB}$ relative to the highest noise level used in Ref. [36] - this combination of signal plus noise remains distinguishable from pure noise as indicated by a standard error a factor of 0.92 smaller than that expected from Eq. (10). Indeed, as $Q$ is a global method, for any $r$ in the chaotic range, $\sigma / \sigma_{\langle Q\rangle}$ approaches unity only when the stochastic contribution tends to infinity, so for any finite noise and a sufficiently long record, it is always possible to detect the presence of chaos. Thus, by sensing and transforming rank fluctuations, $Q$ detects subtle aspects of disorder: the distinction between stochastic noise and deterministic chaos.

\section{A GENERAL (NONLINEAR) REGRESSION PRINCIPLE}

With $Q_{\text {rms }}$, we have a general purpose, indeed with the extension in Sec. XI to general time series, a universal penalty function as an alternative to least squares error. To illustrate this case, we consider the nonlinear parameter estimation problem of fitting two exponential functions. This is well known as an ill-posed problem for a least squares fit. The classical problem in physics for which this model arises is of course radioactive decay. Though we adopt this setting for its familiarity, multiple exponential fits arise in many other arenas, among them the fitting of transmission functions in radiative transfer [39] and dwell-time distributions for ion channels in biophysics [40]. Many special-purpose routines have been written for applications of such multiple exponential fits, and here we consider a representative package, the variable projection method "Varpro" [41], and show that $\min Q_{\text {rms }}$ outperforms it. But, unlike Varpro and other software, e.g., 
implementation of the Padé-Laplace algorithm [42], we change nothing. We minimize $Q_{\text {rms }}$ no differently than we would in fitting a noisy quadratic curve. There are no parameters to tune and no weights [43].

Consider a signal of the form

$$
C(t)=c_{1} \exp \left(\alpha_{1} t\right)+c_{2} \exp \left(\alpha_{2} t\right)
$$

with $c_{1}=1, c_{2}=4$ and $\alpha_{1}=-2, \alpha_{2}=-3$; the observations consist of 50 repeated "measurements" taken at 64 evenly spaced points on the interval $t=[0,1]$. For so short an interval and given relatively close exponents, even the noise-free fitting problem can be challenging. Here, we complicate the situation greatly by the addition of Gaussian noise with $\sigma=3 / 2$. As seen in Fig. 11(a), the raw data show only a general exponential decay; there is no immediate indication of two species. Varpro requires seed values for the exponent pair. Conservatively (to give Varpro a maximum advantage), in all cases, we seed with the exact values. Values for the coefficients and exponents based on $Q$ proceed very much like the earlier process of detrending. One takes initial values for these, substitutes them into Eq. (16), and subtracts the resulting values of $C(t)$ from each of the realizations in the data matrix. The $Q_{\mathrm{rms}}$ of the residual is computed and then minimized by varying the vector of unknown parameters. We used the Nelder-Mead MATLAB routine fminsearch for that minimization.
For the result of the single realization in Fig. 11(a), the $Q$ regression has also been seeded with exact values. While the $Q$ regression does fit the exact result better, the main point about exponential fits is that the Varpro result is a fairly good fit as well. But, where the $Q$ fit yields reasonably accurate coefficients and exponents, the varpro coefficients are wildly in error, of opposite signs, with a meaningless negative value.

In Fig. 11(b), we see the Gaussian pdfs for the standard error of each $Q$-determined exponent, both for $\sigma=3 / 2$ and also $\sigma=1 / 2$. Each of these pdfs is a projection from a fourdimensional pdf. One side effect of that projection is an apparent modest overlap of the two exponents around the value of -2.5 . If one steps back to the two-dimensional pdf projection that is obtained in the $\left(\alpha_{1}, \alpha_{2}\right)$ plane, near coincidence of values becomes a negligible fraction. The sample mean value of $\alpha_{1}-\alpha_{2}$ is 0.95 with a standard deviation of 0.34 , so near equality occurs only at the 3-sigma level. A final revealing (non-normal) pdf is that plotted with dots for $\alpha_{1}$. Here, we show the values obtained with repeated invocations of the Nelder-Mead routine using random perturbations of the starting seeds about their exact values. One does not obtain a single well-defined minimum; rather, there are countless, nearly equal, local minima clustered in a small region leading to a pdf with sample mean of $\mu=-2.049$. From a slice through the 4D volume of $Q_{\mathrm{rms}}\left(c_{1}, c_{2}, \alpha_{1}, \alpha_{2}\right)$, taking, in particular, the $\left(\alpha_{1}, \alpha_{2}\right)$ plane, one sees in Fig. 12(a) a finely structured field with
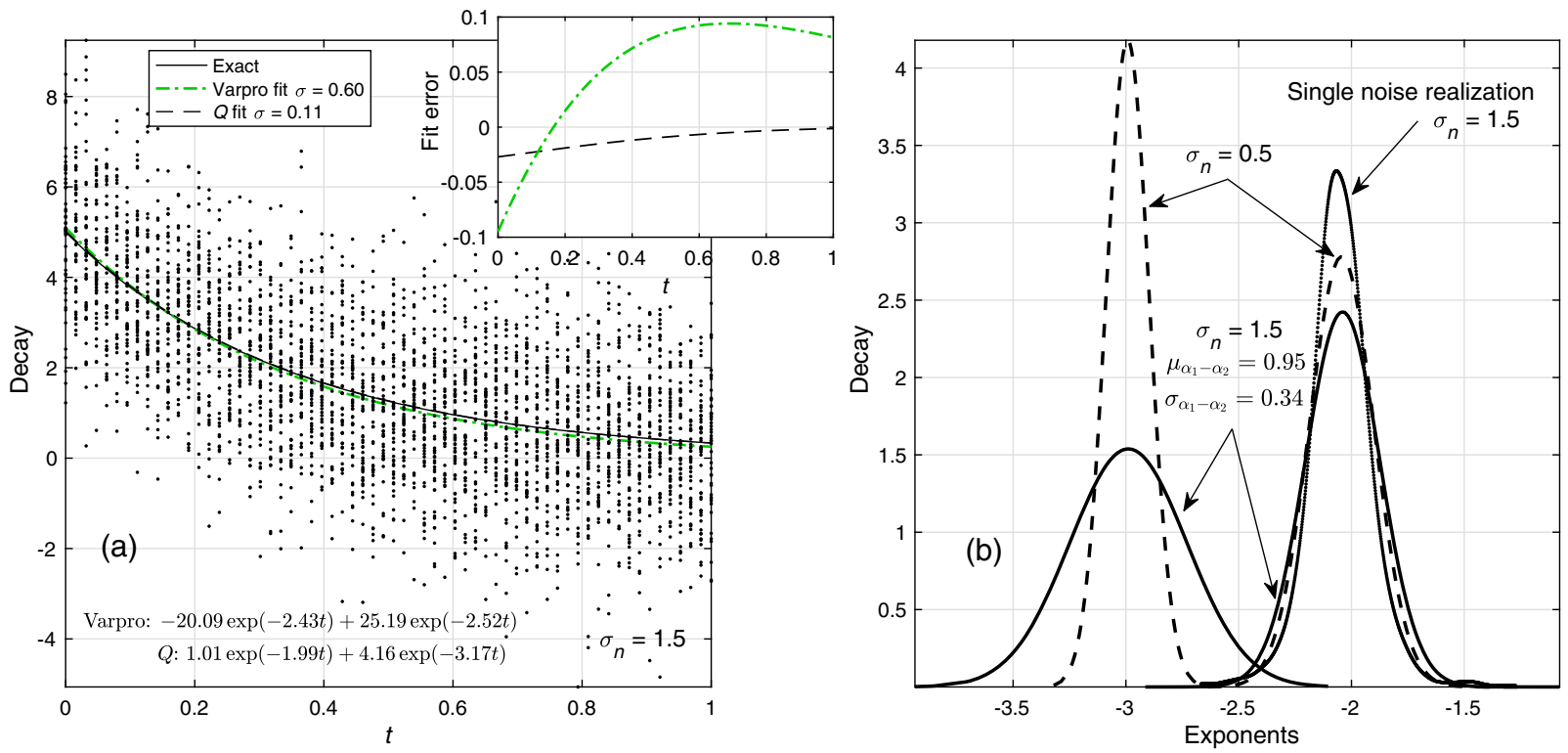

FIG. 11. Nonlinear regression for the two-species radioactive decay. (a) Data are the sum of two decaying exponentials buried in Gaussian white noise with $\sigma=3 / 2$. Also shown are $Q$ and varpro fits and the true signal. The indicated standard errors for $Q$ are relative to the exact answer, and on this basis, the $Q$ regression is 5 times more accurate. But the deeper problem is revealed by the detailed form of the regression for this realization (bottom), where the Varpro result gives two nearly equal exponents and large coefficients of opposing signs, while $Q$ matches all four parameters well. (b) The pdfs for each $Q$ fit exponent from 2500 realizations, solid black line for noise with $\sigma=3 / 2$ and dashed line for $\sigma=1 / 2$. The dotted curve shows the pdf for repeated independent minimizations of a single realization, a consequence of the imbricated surface seen in the next figure. 

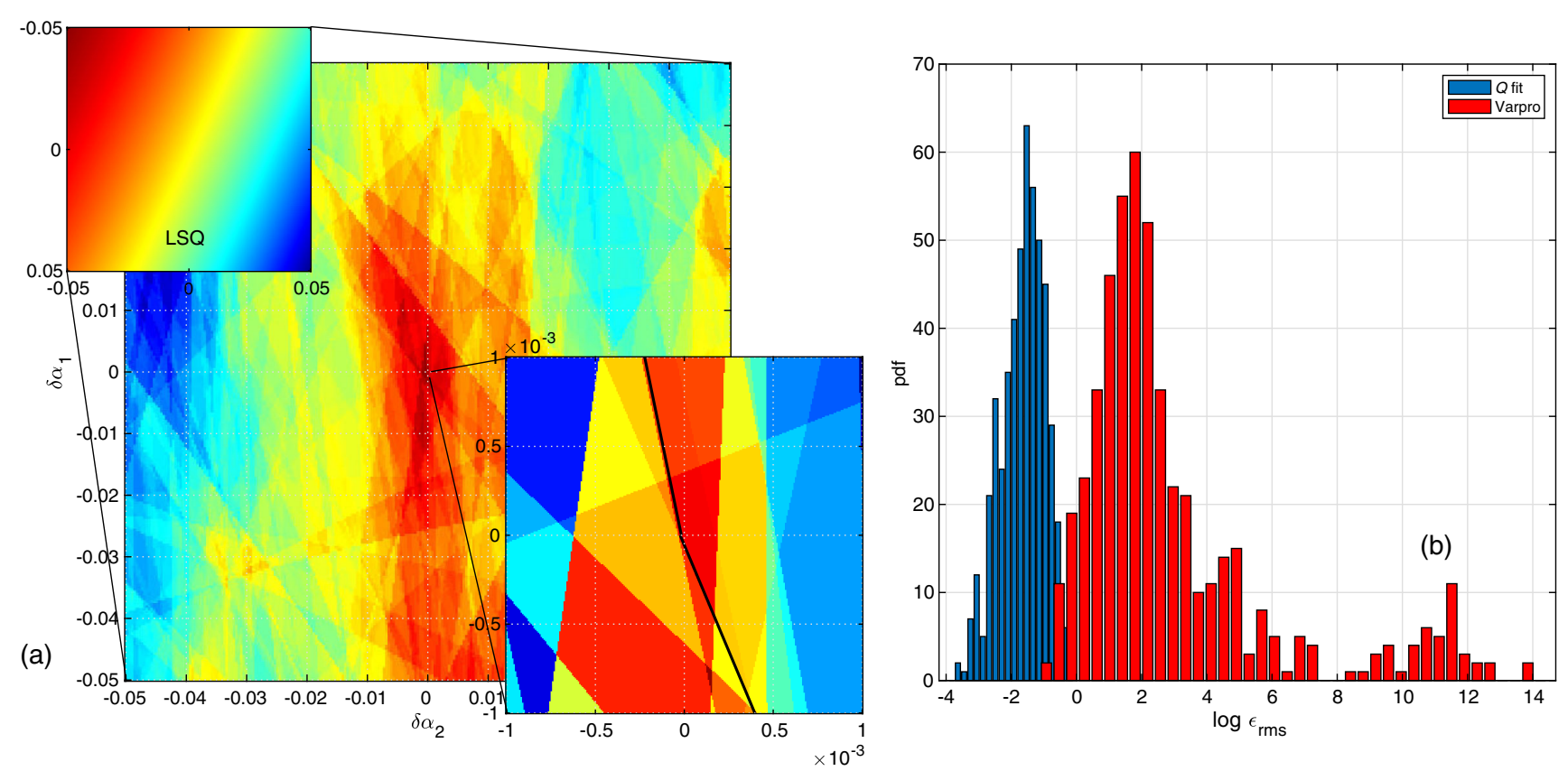

FIG. 12. $Q_{\mathrm{rms}}$ optimization of the decay exponents. (a) The main image shows $1 / Q_{\mathrm{rms}}$ for the single realization above [maxima (red) are more readily visible than minima (blue)], plotted in the $\left(\alpha_{1}, \alpha_{2}\right)$ plane and centered about the exponent pair listed in Fig. 11(a). The discreteness of rank creates this mosaic: a palimpsest of wedges. The Nelder-Mead simplex method is suitable here, but in a given search, it gets trapped by one of many nearly identical local minima. Zooming in around the origin $\times 50$ shows the simplex vertex where the algorithm converged, but almost directly below this point, we see a tiny simplex that (slightly) exceeds this maximum. (Color for the inset is rescaled.) To the upper left is a plot for the entire region of the reciprocal of the conventional LS error, with no local maximum at all. Rather, for this $\left(c_{1}, c_{2}\right)$ pair, the global LS maximum is at $\alpha_{1}=-2.699$ and $\alpha_{2}=-3.055$, significantly worse and far out in the tail of the dotted pdf of Fig. 11. (b) Regarding the unknowns as a four-vector $\mathbf{v}=\left[c_{1}, c_{2}, \alpha_{1}, \alpha_{2}\right]$, with the exact solution denoted by $\mathbf{v}_{0}$, we compute $\epsilon_{\mathrm{rms}} \equiv\left|\mathbf{v}-\mathbf{v}_{0}\right|$ for Varpro and $Q$ fits in a Monte Carlo simulation of 500 trials. Only about $5 \%$ of the former lie to the left of the worst single $Q$ fit.

numerous overlapping wedge-shaped regions. (Maxima are more easily discerned with this color map, so $1 / Q_{\text {rms }}$ is plotted.) Optimization with this simplex structure needs an appropriate routine, and the Nelder-Mead algorithm proves well suited. In the magnified view (inset at lower right), one can see that, while the algorithm has settled on a simplex vertex that is a local maximum, it missed the better, tiny simplex almost directly beneath. These issues are local. All the exponent values for $\alpha_{1}$ found with the randomly perturbed initial seeds are reasonably accurate; moreover, their standard error foreshadows the Monte Carlo simulation with independent realizations of noise. Note that optimization routines customarily allow for user-set tolerances. One of these is the function tolerance: how small a change of $Q_{\text {rms }}$ is realizable. Given that $Q_{\text {rms }}$ derives from rank, this is a discrete value. The smallest possible change is found by perturbing the center of the $P$ matrix with the following $2 \times 2$ matrix:

$$
\left(\begin{array}{ll}
+1 & -1 \\
-1 & +1
\end{array}\right)
$$

This matrix manifestly preserves the row and column sum identities and consists of a rank exchange of one in two adjacent entries. For the model problem here, this leads to
$\Delta Q_{\mathrm{rms}}=4.78 \times 10^{-8}$. Finally, in Fig. 12(b), we compare the Varpro and $Q$ results for the Monte Carlo simulation. The results of the former are so poor that one cannot compare exponent to exponent and coefficient to coefficient. Instead, we adopt a simple gross measure. We let $\mathbf{v}_{0}=$ $\left[c_{1}, c_{2}, \alpha_{1}, \alpha_{2}\right]$ and use $\mathbf{v}$ to denote the vector with components given by their numerically determined values. We then compute $\epsilon_{\mathrm{rms}} \equiv\left|\mathbf{v}-\mathbf{v}_{0}\right|$ as a measure of the error. The dynamic range is so large that we plot the distribution of the $\log$ of this quantity. About $5 \%$ of the Varpro results are slightly better than the single worst $Q$ result and can be sensibly associated with the expected values of coefficients and exponents. About $25 \%$ of the remainder consist of solutions similar to that listed in Fig. 11(a): two nearly equal exponents and coefficients that satisfy $c_{2} \approx 5-c_{1}$, with $c_{1}<0$. For the remaining $75 \%$, one exponent is about -2.5 , and the second is much larger in magnitude. The latter are evenly split between large positive values with coefficients of order $10^{-6}$ and large negative values with coefficients of order one. All of these results, except the initial 5\%, amount to the same conclusion about the data: that there is only a single exponent present.

We have assumed the following: (1) Exponential decay is the correct model, and (2) two species are present. One 
could assume a state of complete ignorance, but we think it fair at least to assume exponential decay is understood to be the relevant model. But, one may not know a priori the number of species [42]. There is then, as a reviewer noted, no basis on which to prefer the varpro or the $Q$ result. As they use different metrics, all one can say is that each has minimized what was asked of it. But there is a difference. Varpro, or any other software that relies upon least squares error for the penalty function, is incapable of stably fitting more than a single exponent for data with this level of noise. The $Q$ fit, by contrast, offers a single-exponent fit of $5.0445 \exp (-2.6552 t)$ and a stable two-exponent fit. However, the values of $Q_{\text {rms }}$ are essentially identical0.021664 (one species) and 0.0216591 (two species)—so one cannot, on that basis, prefer one solution over the other. More evidence is required.

No adaptation is required for the practical application of $Q_{\text {rms }}$ in a multitude of other problems. One simply replaces a routine that computes the least squares error of a trial regression with one that returns $Q_{\text {rms }}$ for the trial. Error bounds are desirable in any application, but one cannot give a universal characterization for these, even for least squares applications. For a linear trend, one can obtain a general form for the standard error of the slope, and this is done for the $Q$ fit in Sec. XI.

\section{SIGNAL EXTRACTION FROM NOISY DATA WITHOUT A PRIORI KNOWLEDGE OF SIGNAL SHAPE}

The opening example in Sec. II established a surprising result: that rank data, lacking all magnitude information, can nonetheless predict linear trends in noisy data, in excellent agreement with slopes found from the traditional (unweighted) least squares. In this section, we argue for a far stronger result: The same rank information yields an assumption-free estimate for a general nonlinear signal with relative amplitude information intact.

Inspired by the close correspondence of undulations in the $\psi_{k}$ modes and oscillations in the companion $\delta \mu_{k}$, we propose that, up to a linear rescaling, the underlying signal is well approximated by $-d \bar{Q}(t) / d t$, where the overbar denotes the mean over rank in $Q$ (i.e., horizontal mean) [44]. The need of linear rescaling arises because rank is invariant under $f(t) \rightarrow \alpha f(t)+\beta$.

Evidently, it is the differential impact of systematic rank arising from signal juxtaposed against random rank scrambling by the noise that allows for the signal magnitude recovery. But this recovery depends upon a finite SNR; the limit of a perfect input signal is singular, and the recovered signal in that limit is (counterintuitively) less accurate.

A sample of which is shown in Fig. 13. The full set consists of $n_{t}=1192 \mathrm{~S}$-wave reflections from the 410- and 660-km mantle discontinuities between 96- and 97-degree epicentral distance. Here, $n_{T}=301$, the data are uniformly spaced at $\Delta t=1 \mathrm{~s}$, and the authors of Ref. [45] use the

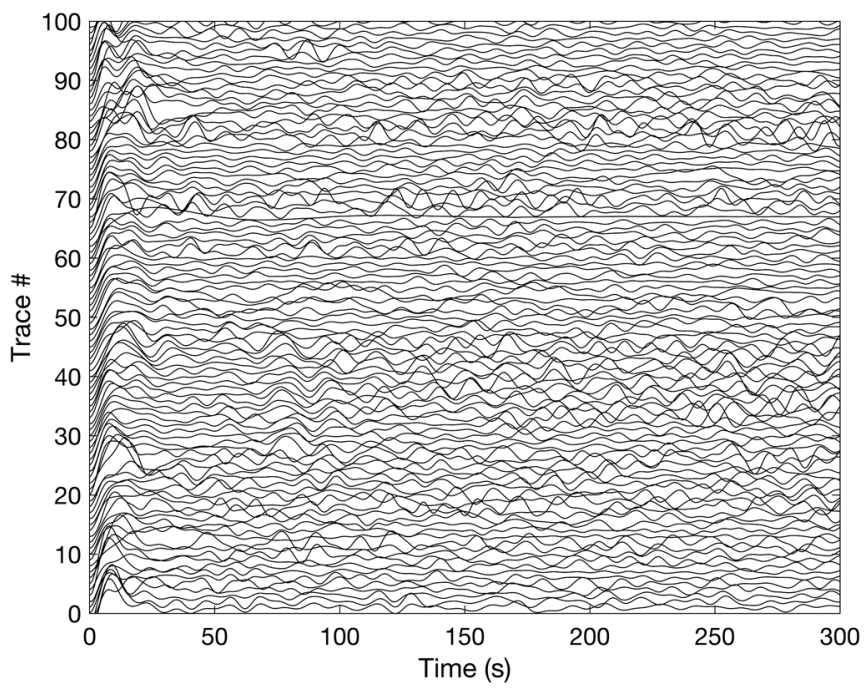

FIG. 13. Raw S-wave seismic wave amplitudes: The first 100 of 1192 traces from the USArray between 96- and 97-degree epicentral distance.

mean over 1192 traces as the signal proxy. In Fig. 14, we compare that signal with the result from $-d \bar{Q} / d t$, with the difference between the two shown in the inset. The two spikes centered at 171 and $255 \mathrm{~s}$ correspond to reflections at the above noted 410- and 660-km mantle discontinuities, respectively. As noted in Ref. [45], the oscillations are part of a signal rather than noise, as these do not decrease as $n_{t}^{-1 / 2}\left(n_{t}=1192\right.$, number of traces), and the $Q$-approach confirms this independently, just as it distinguished chaos from noise in Sec. VII.

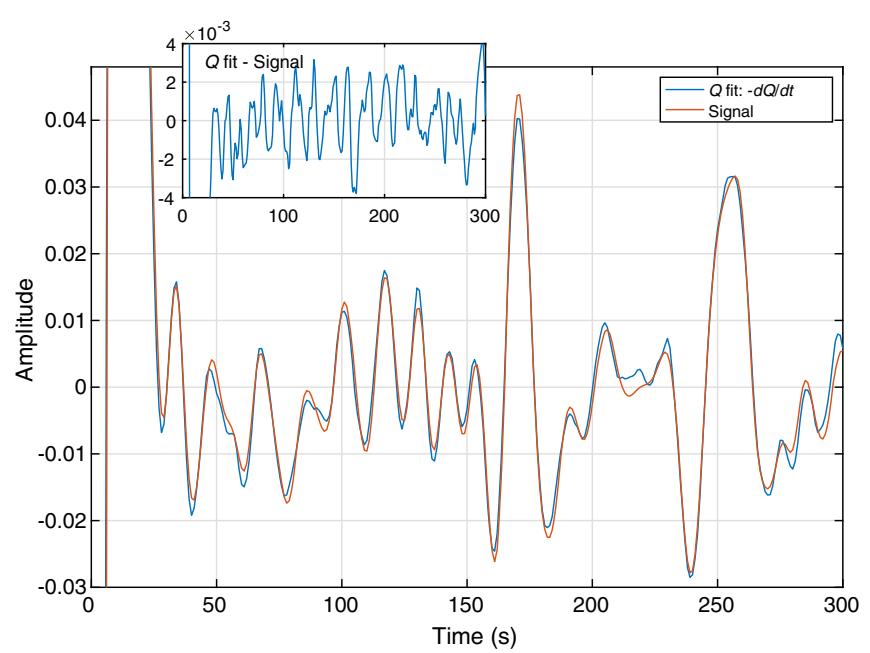

FIG. 14. Signal extraction from $-d \bar{Q}(t) / d t$ : The conventional method (red curve) is to average the noisy individual 1192 traces illustrated in Fig. 13. The resulting S-wave peaks at 171 and $255 \mathrm{~s}$ are linked to mantle discontinuities at 440 and $660 \mathrm{~km}$. The assumption-free, ordinal signal extraction (blue curve) from $-d \bar{Q} / d t$ matches remarkably well, particularly the phase. 
For this comparison, the free linear rescaling of $-d \bar{Q} / d t$ was chosen to match the arithmetic mean most closely. [In a general application without a reference signal, the multiplicative scale $\alpha$ can be set by minimizing $Q_{\mathrm{rms}}(\alpha)$. Here, that dependence is fairly weak, with a shallow minimum that gives a similar result.] The new result from $-d \bar{Q} / d t$ shows excellent fidelity with the benchmark: The phase of all the oscillations is spot on, and the differences are confined to small changes in peak amplitudes.

As with the initial result for Lugano, where we found a slope from annulling $\langle Q\rangle$ of $2.4875^{\circ} \mathrm{C}$ over 65 years, nearly identical to the standard LS fit of $2.5165^{\circ} \mathrm{C}$ per 65 years, here we obtain a result nearly identical to one previously found by more conventional methods. The initial message is, we reiterate, that this agreement is achieved based solely on rank information. Just as for Lugano where we expanded the reach of the $Q$ transform to nonlinear parameter estimation and (in the next section) to data fitting in the presence of heavy tail noise, with results in each case unmatched by conventional methods, here we anticipate that signal extraction with $-d \bar{Q} / d t$ offers comparable opportunities.

\section{X. $Q$ PERFORMS WELL IN HEAVY-TAILED NOISE}

So far, mostly Gaussian white noise has been used, but here we examine distributions with heavy tails, where outliers are ubiquitous. In the least squares family, these are often handled with the bisquare method, which excludes outliers adaptively by assigning them zero weight. However, for distributions with infinite mean and/or variance, a more powerful approach is needed. We are grateful to an anonymous reviewer for suggesting a comparison with the TheilSen estimator, used exclusively to determine linear trends [46]. Its potential limitation is the computation time for large data sets. For example, each trial of $365 \times 64$ data pairs for Table II required $20 \mathrm{~s}$ of CPU time on a 2.5-GHz Intel Core i7 laptop. The full implementation requires fitting slopes to all

TABLE II. Comparison of the standard error for a linear trend with unit rise in 64 years in the following: $Q$, bisquare-weighted robust least squares estimator and, for the two most challenging cases, the Theil-Sen algorithm $\left(10^{3}\right.$ trials). The GEV result for the bisquare least squares has numerous severe outliers for slope estimates. Similarly, for the slightly modified form $x \exp (-x)$ with GEV noise, the bisquare least squares method yields $2.82 \pm 2.81, Q$ gives $1.003 \pm 0.047$, and the Theil-Sen estimator is inapplicable.

\begin{tabular}{lccc}
\hline \hline Distribution & $Q \sigma$ & LS $\sigma$ & Theil-Sen $\sigma$ \\
\hline Uniform $(-1,1)$ & 0.012 & 0.007 & $\ldots$ \\
Gaussian $(\sigma=1, \mu=0)$ & 0.023 & 0.023 & $\ldots$ \\
Cauchy $(\sigma=1, \mu=0)$ & 0.042 & 0.038 & $\ldots$ \\
Pareto $\left(x_{m}=2 / 3, \alpha=2 / 3\right)$ & 0.022 & 0.068 & 0.023 \\
GEV $(\xi=2, \sigma=1, \mu=0)$ & 0.016 & 27.84 & 0.018 \\
\hline \hline
\end{tabular}

possible pairs of points, which takes $\mathcal{O}\left(N^{2}\right)$ operations. Several theoretical papers have proposed $\mathcal{O}(N \log N)$ implementations, but no public code, as far as we know, is available, although CPU time may not be a practical concern for small-to-medium scale applications.

In Table II, the first two cases pose no problem, even for unweighted least squares, though we quote the bisquare result for consistency. The Cauchy distribution is the first point where the bisquare adaptive approach becomes critical; unweighted least squares is not useful. But, then, even the bisquare method begins to lag in performance for the Pareto distribution, until finally it is unusable for the generalized extreme value (GEV) distribution. Both of the latter distributions have infinite mean and variance. By contrast, for Pareto and GEV, Theil-Sen performs admirably as expected, but so does detrending by simply setting $\langle Q\rangle=0$, which is, as noted earlier, already a practical $\mathcal{O}(N \log N)$ algorithm. While the GEV distribution may seem a far-fetched choice, in fact, it arises in applications such as analysis of hydrometeorological data for maximum precipitation events [47].

One can generalize this problem slightly to the multilinear form, $c_{1} x_{1}+c_{2} x_{2}$ [48]. To take a practical example, set $c_{1}=3$ and $c_{2}=-2$ for a $65 \times 90$ grid. For the case of Pareto noise from the $Q$ fit, we obtain $c_{1}=2.9994 \pm$ 0.0425 and $c_{2}=-2.000 \pm 0.0429$. The bisquare algorithm reports $c_{1}=2.9995 \pm 0.0850$ and $-2.000 \pm 0.0772$, so, as in Table II, it is beginning to fray. In contrast, there is no parallel procedure for the Theil-Sen test. There is an work by Dang et al. [49] for the multilinear case, but it remains an unrealized routine for general application. So, for the multilinear case with GEV noise, neither method offers a result to compare with the $Q$ fit of $c_{1}=2.9997 \pm 0.0323$ and $c_{2}=-2.0000 \pm 0.0326$.

Note also that in the general multilinear problem, the $Q$ regression is unusual compared to one's experience based on the least squares formulation. We obtain $c_{1}$ and $c_{2}$ individually by setting $\langle Q\rangle=0$ twice: once for the data matrix in each orientation. This result generalizes to a multilinear form in any number of variables, with the slight modification that one first has to appropriately permute, and then reshape, the matrix for each of the coefficients to be determined. This decomposition is possible because $Q$ is invariant to a constant offset.

\section{GENERAL APPLICATION OF $Q$}

Although the $Q$ transform was developed for regularly spaced data such as those in Fig. 1(a), it is flexible in application, and here we touch upon the possibilities. For example, uniform spacing of the temperature data by day and year could be replaced by recording daily low temperature when first attained, i.e., by the continuous astronomical Julian date, including the hour, minute, and second. Then, the abscissae are irregularly spaced. 

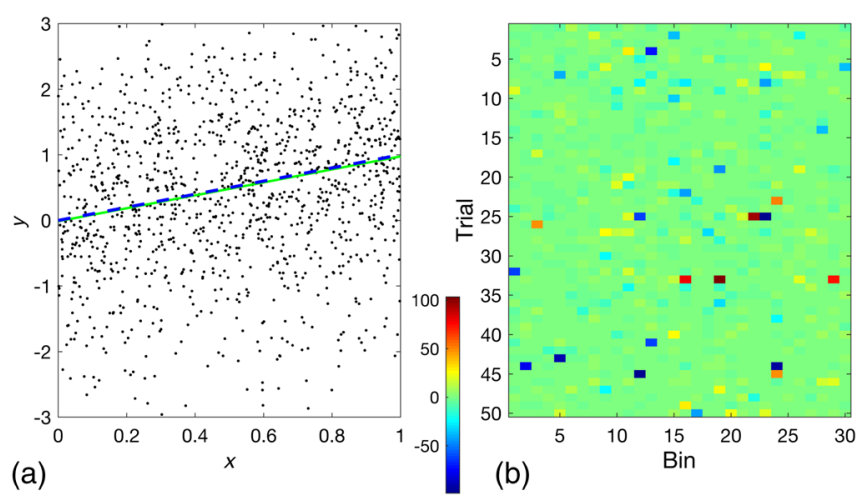

(b)

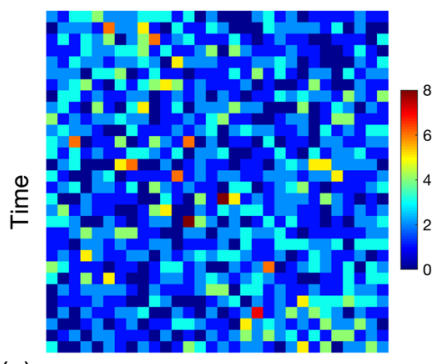

(c)

Rank

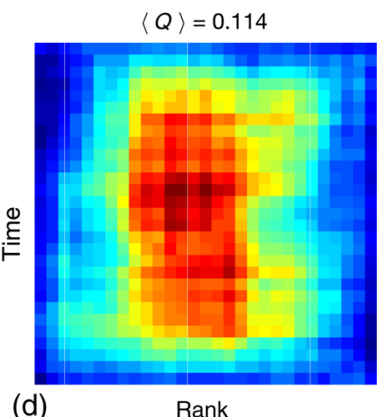

(d)

Rank

FIG. 15. Application of $Q$ to general data fitting. (a) A sample of 1500 data points ( $x$ values) drawn from a uniform distribution. The $y$ values show a linear trend plus noise from a Cauchy distribution. The vertical range is truncated to show the local fit, but the actual data range over $[-100,100]$. (b) While randomly space sample points seem far from the initial application of linear regression with $Q$, by suitably grouping these points, we obtain the equivalent of Fig. 1(a). Panel (c) shows the $P$ matrix and panel (d) the $Q$ matrix. Here, $Q$ and, more so, $P$ clearly show the imposed linear trend. The $Q$ fit trend (solid line), supplemented by a local algorithm to estimate the constant term, compares well with the exact trend (dashed line).

A model data set is plotted in Fig. 15(a), consisting of $1500\left(x_{k}, y_{k}\right)$ pairs. The $x_{k}$ coordinates are generated from a uniform distribution on the interval $[0,1]$. The $y_{k}$ values are given by

$$
y_{k}=x_{k}+n_{k},
$$

where $n_{k}$ are noise values from a Cauchy distribution with mean $\mu=0$ and scale $\sigma=1$.

We need a data matrix from which to compute $P$ and $Q$. For this purpose, we subdivide the $x_{k}$ into $M$ bins each with $N$ points, with $M \times N=1500$. We choose comparable $M=50$ and $N=30$. From the asymptotic formula in Eq. (10), to leading order, there is no difference if these are reversed, and the numerics confirm it. While Eq. (10) is asymptotic in $n_{T}$, and breaks down as $n_{T}$ becomes small, $n_{t}=1$ is permitted (although $Q$ itself is then much larger). Note that $M$ should be chosen with the Nyquist frequency in mind whenever information about the expected signal is available.
Now, we partition the $x$ data into the 30 bins: the first bin containing the 50 smallest values of $x, \ldots$, up to the last bin with the 50 largest. Each $x_{k}$ is then paired with its corresponding $y_{k}$, so bin \#1 now contains $50(x, y)$ pairs, and so on. Finally, we assemble the 50 trials. For experiment 1 , take element $1\left(y_{1}\right)$ from bin \#1, element $1\left(y_{51}\right)$ from bin \#2, etc. Up to the 50th experiment: Take the last remaining element from bin \#1 $\left(y_{50}\right)$, etc.

The resulting data matrix is shown in Fig. 15(b), along with a color scale to show the wide range of values associated with this noise distribution. From one row to the next, the $x$ coordinate in a given column is no longer constant (previously the calendar year), but, as each experiment is independent, nothing hinges upon that constancy; each row still represents a linear trend that we attempt to estimate as a function of the horizontal coordinate. For the purposes of computing $P$ and $Q$, that horizontal coordinate is the (integer) bin number, while if a specific functional relation (trend) is to be tested, we appeal to the specific $x_{k}$ for that row and column.

Proceeding to enumeration of $P$, we obtain the $30 \times 30$ matrix illustrated in Fig. 15(c). Although noisy, the $P$ matrix has a bias, with the upper left and lower right overpopulated, indicating a positive trend. The $Q$ matrix on the right confirms this. Here, $\langle Q\rangle=0.114$, and this result can be compared to the noise benchmark in Eq. (10) on the assumption that the latter does indeed hold for all distributions. For the present $M$ and $N$, one obtains $\sigma_{\langle Q\rangle}=0.0196$, and $\langle Q\rangle$ here is well above the noise level; there is a signal. Moreover, $Q_{\mathrm{rms}}=0.1395$, and the ratio $\left|\langle Q\rangle / Q_{\mathrm{rms}}\right|=0.8193$ is very close to the ratio of 0.8341 for a pure linear signal with no noise, evaluated at 30 points, indicating that the signal, based on $Q_{\mathrm{rms}}$, has nearly the maximum trend possible based on $\langle Q\rangle$.

We annul $\langle Q\rangle$ exactly as before, making sure that, when the trend is computed, matrix entries are computed individually since columns of the raw data matrix are no longer at fixed $x$. The result is a slope estimate of 0.9869 , hence an error of 0.0130 . The result is plotted as a solid line in Fig. 15; the exact result is shown as a dashed line.

The generalized $Q$ fits are insensitive to a constant offset, and one has to find another tool for that purpose. For heavytailed distributions, a local method of matching the estimated sample is preferable. On mild assumptions about noise statistics, one expects a fitted line to lie in the dense "middle" of a cloud of sample points. A simple method to estimate that middle is first to subtract the $Q$ fit and then to count the sample points lying within a sliding window, fixing the intercept as the midpoint of the window location where the convolution peaks. We used the first member of a Slepian sequence $[50,51]$ for that window successfully, yielding the intercept for the solid line in Fig. 15(a).

This exercise, repeated 1500 times, gives an estimate for the mean slope error of 0.0060 , consistent with a limiting value of zero, that is, an unbiased estimator. It also gives an 
estimate for the standard deviation of the slope of 0.171 . For the latter, note that

$$
\frac{\sqrt{30 \times 50}}{\sqrt{64 \times 365}} 0.171=0.043
$$

consistent with the value of 0.042 reported in Table II.

\section{A. Data matrix considerations and error estimates}

We have constructed raw data matrices reflecting a variety of origins of noise and signal. The simplest circumstance is $\delta$-correlated noise, with successive rows representing $n_{t}$ repeated trials and convergence to the underlying signal scaling as $n_{t}^{-1 / 2}$. Typically, $n_{T}$ would be determined by the expected signal duration or period. In most instances, either $\langle Q\rangle$ or $Q_{\text {rms }}$ would serve as the metric, and their general asymptotic expansions are as indicated in Eqs. (10) and (12). Beyond this, from Eqs. (B1) and (B2), we have the analytic foundation to demonstrate that for such noise in the absence of signal, the ensemble average $P$ is constant and hence $Q$ vanishes.

Often, however, the noise is stationary but correlated. As shown in Appendix B 1, the ensemble average of $P$ is no longer constant, and hence the mean $Q$ is nonzero. But, since the induced $P$ has $D_{4}$ symmetry, the ensemble mean of $\langle Q\rangle$ is still zero. One must still compute the modified standard deviation to set appropriate thresholds for signal detection. While the mean of Eq. (10) is altered by the $D_{4}$ corner effects on $P$, this diagnostic has a nonzero mean in all cases, so Monte Carlo computations will automatically adjust for this correction. Still, in some cases, it may make sense to use an altered $\tilde{Q}_{\text {rms }}$ based on $\tilde{P}$, with its $D_{4}$ projection removed.

The raw data matrix in the introductory climate example manifests another phenomenon. Here, the data were wrapped vertically in the matrix; that is, the end of column 1 then continues at the top of column 2, and so on. Not only do we have the vertical correlation whose effects were considered in Sec. IV, but, from the theoretical perspective of Appendix B 1, one would also need to model the crosscorrelation between columns. Therefore, we must rely upon numerical evidence even more. As first noted in Sec. II, the principal effect of (positive) vertical correlation is a reduction in the effective value of $n_{t}$. Extensive numerical simulation further indicates that, in spite of cross-column correlation, the ensemble mean $P$ remains constant in the absence of signal provided the columns are long enough, relative to the correlation length, so that row elements are uncorrelated.

A variant of this issue arises if one seeks to extract not the long term but the seasonal signal. Then, the original data matrix for Lugano is turned 90 degrees, so it is the end of one row that is correlated with the start of the next. Again, the ensemble mean of $P$ for pure noise is not constant, so the ensemble mean of $Q$ is not zero. While this again represents a potential bias, numerical results suggest that the ensemble mean $P$ still has $D_{4}$ symmetry and hence does not affect estimated trends in the mean, only estimated trends in variance [52].

With this preamble, we turn to the practically important question of error analysis in the simplest case of iid noise. As remarked previously, when looking for estimated slope error, one has to restore the link between the rank-order space of $Q$ and the dimensional space of the raw data. Now, the underlying pdf of white noise-Gaussian, Cauchy, etc.-affects the slope error. If the noise in Eq. (17) is replaced by Gaussian noise with standard deviation $\sigma$, then for $K=M \times N$ total sample points, the large $K$ limit of the standard deviation of the (unit) slope for an unweighted least squares fit is

$$
\sqrt{\frac{12}{K}} \sigma \approx \frac{3.46 \sigma}{\sqrt{K}}
$$

For the present procedure, we can appeal to the leading term of Eq. (10), which must then be divided by the ensemble average of $d\langle Q\rangle / d \alpha$ evaluated at $\alpha=0$ to calibrate the change in the mean value of $Q$ when perturbed by a signal $\alpha x$. It is through this factor that the connection between the particular noise distribution and signal manifests itself, accounting for the variation of the $Q$ entries in Table II. In principle, this derivative can be computed analytically by taking the mean value of the $Q$ transform of the Fréchet derivative of Eq. (B2). Short of that, direct numerical evaluation of that Fréchet derivative for $M=15$ leads to $d\langle Q\rangle / d \alpha=0.2422 / \sigma$. This result can be compared in a test of consistency to a numerical fit from Monte Carlo simulations for varying $M$ of

$$
\frac{1}{\sigma}[0.2117+0.1618 \exp (-0.1087 M)]
$$

which gives $0.2434 / \sigma$ at $M=15$. Taking the large $M$ limit of Eq. (19) and the leading term in Eq. (10) then gives the standard deviation of the slope estimate as

$$
\frac{0.7131 \sigma}{0.2117 \sqrt{K}}=\frac{3.37 \sigma}{\sqrt{K}} .
$$

Hence, LS and $Q$ fits of slope are, for this Gaussian case, essentially identical. As noted in the Introduction, LS is the maximum likelihood estimator for this case; hence, one cannot improve upon Eq. (18). That the constant in Eq. (20) is slightly smaller is not, however, a contradiction. Rather, it reflects a compounding of errors from two delicate estimations for asymptotic constants, namely, Eqs. (19) and (10).

Strictly speaking, Eq. (10) only applies for a discrete set of $n_{T}$ abscissae, not to the larger generalized set of $K$ points 
here. But for the above estimate, we need only a leadingorder result, and for that it suffices to use Eq. (10) with the abscissae chosen as the column-by-column means.

The procedure above extends readily to fitting an unknown signal by minimizing $Q_{\mathrm{rms}}$ using an expansion in a basis set of the user's choosing. One can extend the binning here to higher dimensions and then parallel the development of Sec. VIII. Lastly, one can pursue the second half of the $Q$ formalism, with $-d \bar{Q} / d t$, but this is beyond the scope of this paper.

\section{CONCLUDING REMARKS}

The unknown signal, whether deterministic or random, is defined here by the departure from the "equality of ranks," that is, uniformity (constancy) of the ensemble-averaged rank population matrix $P$. At a single realization level, the departure is from the salt-and-pepper $P$ (Poisson process). The logic is reminiscent of the first law of thermodynamics: When introducing internal energy, one does not yet know what "heat" is, but one understands its absence through heat insulation. Remarkably, this "not noise" definition readily distinguishes deterministic chaos from noise as illustrated on the data produced by the logistic map even in the presence of significant white noise. That same fingerprint which, for some, serves to detect signal can, for others, serve to revise the noise threshold against which some other signal is then judged.

The ordinal nature of the $Q$ transform introduced in this paper gives it great versatility: It extends to time series with different units and to imaging. It is robust with respect to gaps in data. The algorithm is simple, objective, and fast. It performs well in various types of noise, including heavytailed noise.

A central achievement of Sec. V is the precise disentanglement of sample variability in the mean from that in the variance. This disentanglement is accomplished by a surprising connection between heretofore disparate fields: random process theory and group theory. In particular, we employ symmetry arguments in the rank-order $Q$ plane where sample variability in the mean is shown to be paired uniquely with the dihedral symmetry $D_{4}$.

Fitting of several exponentials (Sec. VIII) is widely known as an ill-posed problem in nonlinear least squares, and no other method known to us can provide a robust solution for two exponents with noise of the magnitude used here. The ill-posedness for the least squares is rooted in the near singularity of the Hessian matrix. Similar illposedness applies to other (nonexponential) nonlinear parameter estimation problems as well. The robustness of the $Q$ transform is due to a rank-based metric objective function in lieu of least squares error.

For linear regression in heavy-tail noise, the state of the art is well represented in Ref. [46] with the weighted TheilSen method. While weighting mildly improves the performance of classical Theil-Sen, there is no viable algorithm faster than $\mathcal{O}\left(N^{2}\right)$ operations. For large data sets, this can become prohibitive. Our method compares favorably with Theil-Sen and takes only $\mathcal{O}(N \log N)$ operations.

Moreover, while the Theil-Sen algorithm is limited to linear regression, the $Q$ transform is free of any restriction on functional form. In this paper, for brevity, we indicate only an initial extension to the case of a multilinear fit. In principle, the Theil-Sen algorithm could extend to this case as well, albeit at a huge expense in operations count. For any nonlinear functional form, even only of one variable, there are no other general methods.

Often it is desirable not to prejudice the process of signal extraction by imposing a specific structure, e.g., polynomial, trigonometric, etc. One wishes instead for an assumption-free means. A principal family for this purpose is the family of moving average and weighted moving average methods. But these methods perform poorly in the presence of nonstationary variance or intermittent outliers. The $Q$-transform method of signal extraction is free of such limitations.

We hope that the reader will try these ideas, as the MATLAB code is supplied in the Supplemental Material [53].

\section{ACKNOWLEDGMENTS}

This work was supported by NSF Grant No. AGS1639868. We thank anonymous reviewers for suggesting consideration of the Theil-Sen algorithm, the logistic map, and the Ornstein-Uhlenbeck process.

\section{APPENDIX A: DETECTING BREAKS IN A TIME SERIES}

Another, more quantitative prediction follows from Eq. (3) by noting that once a linear trend is removed from a data set, the residual $Q$ is often a double-lobed horizontal structure of alternating sign. This is the signature of a correction to the temperature profile with alternate periods of cooling and warming, but no net trend. Similar bimodal patterns arise in $Q$ after detrending either a quadratic temperature profile or a piecewise linear version but with significant differences, as shown in Fig. 16.

A simple algebraic representation of $P$ for the piecewise case may be taken as a trendless, zero mean, piecewise linear profile in $y$ with a node at $y_{n}$, multiplied by $x$. Application of Eq. (5) then yields

$$
\begin{aligned}
q(x, y)= & \frac{1-x^{2}}{2\left(y_{n}+1\right)\left(y_{n}+2\right)\left(y_{n}-1\right)^{2}\left(1-y^{2} x^{2}\right)} \\
& \times\left[2\left(y-y_{n}\right)^{2}\left(H\left(y_{n}-y\right)-H\left(y-y_{n}\right)\right)\right. \\
& \left.+y^{2} y_{n}^{3}-3 y^{2} y_{n}+2 y y_{n}^{2}-y_{n}^{3}+2 y-y_{n}\right] .
\end{aligned}
$$

A typical pattern for Eq. (A1) is seen at the top left in Fig. 17. To the right is the residual $Q$ after removing the linear trend for station USW00023050 (Albuquerque 


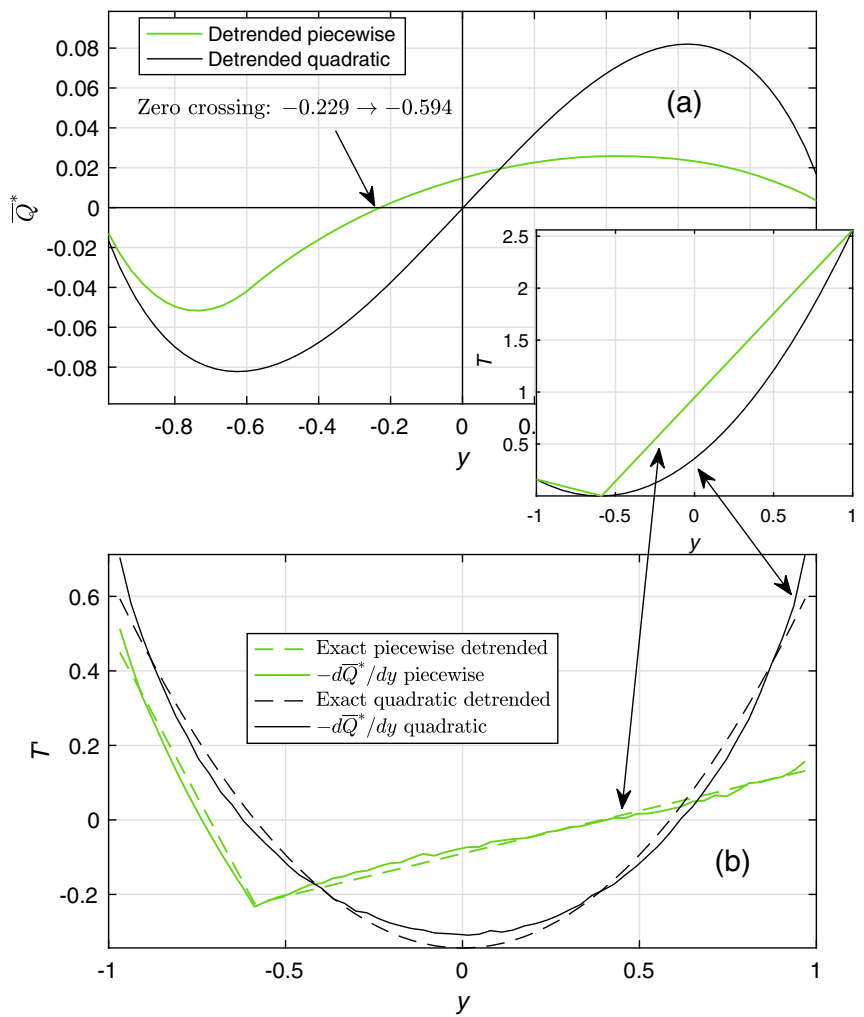

FIG. 16. Quadratic vs piecewise linear profiles and break detection: Two profiles are shown in the inset: the quadratic, $(y+3 / 5)^{2}$, and a piecewise linear profile matching the endpoint values and zero minimum at $y=-3 / 5$. (a) Ensemble means $\bar{Q}^{*}$ computed for iid normal noise with $\sigma=2$. The antisymmetric cubic profile is consistent with the detrended quadratic, which is centered at the origin, while the zero crossing of $\bar{Q}^{*}$ for the detrended piecewise case maps accurately using Eq. (A2) to $-3 / 5$. (b) $-d \bar{Q}^{*} / d y$, when linearly rescaled, works well for both detrended profiles. If a bimodal pattern of $Q$ emerges after detrending with a zero crossing of $\bar{Q}$ significantly displaced from the middle, this is, likely, a discontinuity in the time series at the indicated node (as inferred in Fig. 11), caused by, e.g., changes in thermometry or station location. In such cases, relying on $-d \bar{Q} / d t$ from a single realization is less robust than using Eq. (A2), with no differentiation to fix the node. (A significant cubic component in the profile can also displace the zero crossing, but this typically shows in the annual mean.)

International Airport, NM). The dashed line is a zero contour of $Q$ on the left, chosen to coincide with the zero of the horizontal mean of the $Q$ at the right.

A self-consistent way to achieve a breakpoint is to simultaneously detrend each of $Q_{L}, Q_{R}$, and $Q$ as well as possible, while requiring that the trend used for the third one be the net slope of left and right segments joined as a continuous function. This imposes a jump condition. Each of the three mean values for $Q$ is first weighted by $\sqrt{n_{T}}$ to put them on an equal footing, and their sum of squares is then minimized. The dash-dotted line in Fig. 17 shows the (a)
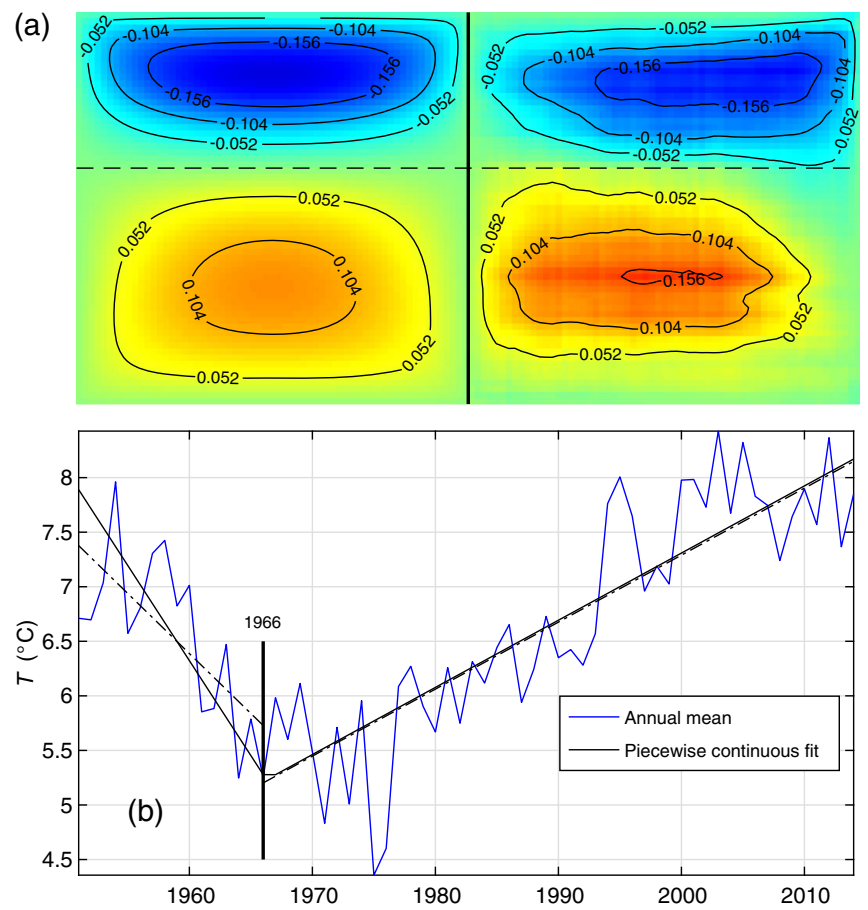

FIG. 17. Inference from $Q$ of a break in slope. (a) The slight left-right asymmetry in the pattern at the top right (USW00023050 Albuquerque International Airport, NM) is a harbinger of mode 2 in Fig. 6, with a downward trend in $\sigma$ of about $0.4^{\circ} \mathrm{C}$. (b) $Q$ and LS detrending of the left-hand segment alone give a different slope than results from this piecewise continuous correction, thereby suggesting a breakpoint.

optimal result. The continuity constraint yields a jump of $0.53{ }^{\circ} \mathrm{C}$ for raw temperatures to the left of the break. We note a similar isolated nearby empirical breakpoint in 1961, with an estimated bias of $0.5^{\circ} \mathrm{C}$ to the left, for the monthly mean data for this station in the Berkeley Earth series (\#173069). While the coincidence of the bias estimates is striking, the onset date here has to be refined since the deduction based on Eq. (A2) assumes a piecewise continuous profile. A simple trial confirms that a jump moves $y_{n}$ earlier.

From Eq. (A1), the exact general result is as follows:

$$
y_{0}=\frac{y_{n}}{2+\left|y_{n}\right|}
$$

where $y_{0}$ is the zero line of $\bar{Q}$. It follows then that all zeros of a bimodal $Q$ must always lie in the middle one-third of the domain. For a time span of 64 years, that amounts to the middle 21. From a sample of 79 GHCN stations with unbroken temperature records, 40 exhibited an evident bimodal pattern after detrending. In all cases, the zero of the horizontal average of the residual $Q$ observes this constraint; moreover, the relation above then furnishes an objective location for the break point in a piecewise temperature correction, leaving only its amplitude to be 
determined. For the case illustrated, the zero of $Q$ is at the beginning of 1976, in accordance with the middle-third rule, and the indicated node is hence early in 1966.

\section{APPENDIX B: MORE ON ANALYTIC RESULTS}

\section{An expression for the ensemble mean of $P$}

To gain a deeper understanding of $Q$ transform properties (e.g., the signal extraction conjecture $-d \bar{Q}^{*} / d t$ or normality of the distribution for $\langle Q\rangle$, discussed in later sections), we note here an exact general result for the ensemble mean of $P$ in the case of uncorrelated iid variables with a secular component $T_{k}$ ( $k$ is a year index from 1 to $K$ ), namely,

$$
\begin{aligned}
P_{n, k}(T)= & n_{t} \int_{a}^{b} d t \operatorname{pdf}\left(t-T_{n}\right) \sum_{j=1}^{K-1} \prod_{n=1}^{C_{k-1}} \operatorname{cdf}\left(t-T_{j} s_{n}\right) \\
& \times \prod_{m=1}^{K-k}\left(1-\operatorname{cdf}\left(t-T_{j}\right)\right)
\end{aligned}
$$

where pdf and cdf are the governing probability density and cumulative distribution functions on the interval $[a, b]$ with appropriate parameters as needed. Here, ${ }_{K-1} C_{k-1}$ is the binomial coefficient, $s$ is a matrix whose rows contain all possible choices of $k-1$ elements from the set $\{1,2, \ldots, K\}_{\underline{n}}$ and

$$
\left\{{ }_{j} \tilde{s}_{m}\right\} \equiv\{1,2, \ldots, K\}_{\underline{n}}-\left\{{ }_{j} s_{n}\right\} .
$$

For the useful particular case of Gaussian random components with standard deviation $1 / \sqrt{2 \beta}$, the $(n, k)$ element of $P$ is given by

$$
\begin{aligned}
P_{n, k}(\beta \mid T)= & n_{t} \sqrt{\frac{\beta}{\pi}}\left(\frac{1}{2}\right)^{K-1} \int_{-\infty}^{\infty} d t \exp \left(-\beta\left(t-T_{n}\right)^{2}\right) \\
& \times \sum_{j=1}^{K-1} \prod_{n=1}^{C_{k-1}}\left(1+\operatorname{erf}\left(\sqrt{\beta}\left(t-T_{j^{s_{n}}}\right)\right)\right) \\
& \times \prod_{m=1}^{K-k}\left(1-\operatorname{erf}\left(\sqrt{\beta}\left(t-T_{j^{\tilde{s}_{m}}}\right)\right)\right) .
\end{aligned}
$$

A test of this prediction for a linear $T$ against the mean from Monte Carlo trials with $N$ realizations gives a residual with rms error that decays as expected, like $N^{-1 / 2}$. The Fréchet derivative of these forms proves a central ingredient in error bounds for linear regression. We return to this point in Sec. XI, where the $Q$ transform is broadened to general time series. It would be useful to generalize the equilibrium form (B1) to correlated noise, but even the uncorrelated Gaussian case in Eq. (B2) is difficult, e.g., proving that $P_{n, k}(\beta \mid 0)=n_{t} / K$ in the absence of any signal is a complex task of integration and combinatorial identities. Moreover, as it stands, owing to the factorial growth of terms, Eqs. (B1) and (B2) are computationally feasible only out to $K \approx 16$, smaller than needed in practice. An asymptotic expansion is needed.

\section{Effects of correlation: End effects on $P$}

Even for a stationary random process, correlation introduces a surprise: The ensemble average of $P$ is no longer constant. One can see the origin of this by considering a time series of exactly three entries, $[x, y, z]$. If these are iid with the standard normal distribution (zero mean, unit variance), then the joint pdf for this set is given by

$$
p_{1}(x, y, z)=\frac{1}{4 \sqrt{2 \pi^{3}}} \exp \left(-\left(x^{2}+y^{2}+z^{2}\right) / 2\right) .
$$

From the symmetry of this form alone, it follows that the probability for each variable being the lowest rank is $1 / 3$. Numerical experiments suggest that this conclusion holds for any iid distribution, a result that may be strengthened by appealing to the argument in Ref. [13], which notes that reshuffling records destroys any rank correlation in a time series.

We introduce correlation in the simplest possible fashion. Let $x=x_{1}+x_{2}, y=x_{2}+x_{3}$, and $z=x_{3}+x_{4}$, where $x_{1,2,3,4}$ are iid normal variables as above. Now, $x$ is correlated with $y$, and $y$ with $z$, but $x$ and $y$ are uncorrelated. Then, the joint pdf is

$$
\begin{aligned}
p_{2}(x, y, z)= & \frac{1}{4 \sqrt{2 \pi^{3}}} \exp \left(-y^{2} / 2-3\left(x^{2}+z^{2}\right) / 8\right. \\
& +y(x+z) / 2-x z / 4)
\end{aligned}
$$

where

$$
\begin{aligned}
& \int_{-\infty}^{\infty} d x p_{2}(x, y, z)=\frac{1}{2 \pi \sqrt{3}} \exp \left(\left(y z-y^{2}-z^{2}\right) / 3\right), \\
& \int_{-\infty}^{\infty} d z p_{2}(x, y, z)=\frac{1}{2 \pi \sqrt{3}} \exp \left(\left(x y-x^{2}-y^{2}\right) / 3\right), \\
& \int_{-\infty}^{\infty} d y p_{2}(x, y, z)=\frac{1}{4 \pi} \exp \left(-\left(x^{2}+z^{2}\right) / 4\right)
\end{aligned}
$$

and one sees, in the last three forms, the correlation relations stated above. Now, the computation for the lowest rank yields 


$$
\begin{aligned}
& \int_{y=x}^{\infty} \int_{z=x}^{\infty} \int_{x=-\infty}^{\infty} p_{2}(x, y, z) d x d y d z \\
& =\int_{y=z}^{\infty} \int_{x=z}^{\infty} \int_{z=-\infty}^{\infty} p_{2}(x, y, z) d x d y d z=3 / 8
\end{aligned}
$$

and

$$
\int_{z=y}^{\infty} \int_{x=y}^{\infty} \int_{y=-\infty}^{\infty} p_{2}(x, y, z) d x d y d z=1 / 4
$$

with an overshoot at the ends and a low in the middle. The symmetry breaking here is that $y$ is correlated with two neighbors, $x$ and $z$ with only one. For an extended row of this same construction, that symmetry breaking remains confined to the ends. A similar result is obtained for the highest rank.

In consequence, for correlated stationary noise, all four corner regions of $P$ are affected, while the interior approaches constancy. For progressively larger $P$, the fractional area affected tends to zero and so does the induced ensemble average of $Q$. This effect manifests as a pure $D_{4}$ contribution to $P$ and pure $R_{2}$ for $Q$ and so leaves trends completely unaffected. In cases where a variance signal is sought, one could first simulate the noise in a Monte Carlo computation, obtain the ensemble average $P$, and then remove its zero-mean projection on all realizations with the variance signal present.

\section{Analysis for asymptotics of $\langle Q\rangle$}

While a derivation of Eq. (10) is challenging, one can approach it with a simplified model developed from a computationally efficient observation about Eq. (2). Noting the earlier recasting of $\mathbf{q}=M \mathbf{p}$, if one is solely interested in $\langle Q\rangle$, this is obtained by left multiplying on both sides by $\mathbf{1}^{T}$, a row vector of ones. We can premultiply on the right, denoting the result as $\mathbf{m}^{\mathrm{T}}=\mathbf{1}^{\mathrm{T}} M$. The result for $\langle Q\rangle$ requires $K=n_{T} \times n_{t}$ flops, and the computation is dominated by $n_{T} n_{t} \log n_{T}$ flops for the sorting operation needed for $P$. It is instructive to reconstitute $\mathbf{m}$ as a matrix, as shown in Fig. 18 [54]. The sum of the pointwise (Hadamard) product of this field with the noisy data in $P$ is the precise content expressed in $\langle Q\rangle$, as is the meaning of $\langle Q\rangle=0$. Recall that the entries in $P$ are correlated Poisson random variables. Specifically, to leading order, any element $p_{i, j}$ has a correlation of $-1 / n_{T}$ with all other elements in the $i$ th row and $j$ th column. For typical values of $n_{T}$, this is a weak correlation, so we consider instead a companion matrix $\tilde{P}$ populated by uncorrelated Poisson variables with the same parameter, $\lambda=n_{t} / n_{T}$. Half the elements in $\mathbf{m}$ are positive; the other half are the negatives of these. Accordingly, we partition the contraction $\mathbf{m}^{\mathrm{T}} \tilde{\mathbf{p}}$ into the corresponding contributions. We can use a normal approximation for the sum of uncorrelated Poisson variables with positive-definite coefficients. The variance of the resulting normal random variable is

$$
\frac{n_{t}}{n_{T}} \sum_{k=1}^{\left\lfloor n_{T}^{2} / 2\right\rfloor}\left(m_{k}^{(+)}\right)^{2}
$$

A second normal random variable from the sum with negative coefficients has exactly the same variance. Consequently, the variance of the final sum of these two is twice the above. (The means of the two are equal and opposite, so the mean of their sum is zero.) The standard deviation then follows directly. The elements $m_{k}^{( \pm)}$could be expressed exactly by reference to Eq. (1), but the algebra would be formidable, to say nothing of the sum. But one can observe that $m_{k}^{( \pm)}$depends solely upon $n_{T}$, save for the overall prefactor of $1 / n_{t}$. Here, the asymptotic result that follows is

$$
\sigma_{\langle\tilde{Q}\rangle} \sim \frac{0.7015}{n_{t}^{1 / 2}}\left[\frac{1}{n_{T}^{1 / 2}}+\frac{2.0313}{n_{T}^{3 / 2}}+\mathcal{O}\left(\frac{1}{n_{T}^{5 / 2}}\right)\right]
$$

With less than a $2 \%$ change in the leading-order coefficient, this result is very close to Eq. (10). The main distinction is the absence of a term of order $1 / n_{T}$. Such a term cannot arise from the algebra that generates $m_{k}^{( \pm)}$. Rather, it stems from the weak correlation of order $-1 / n_{T}$ for the full problem.

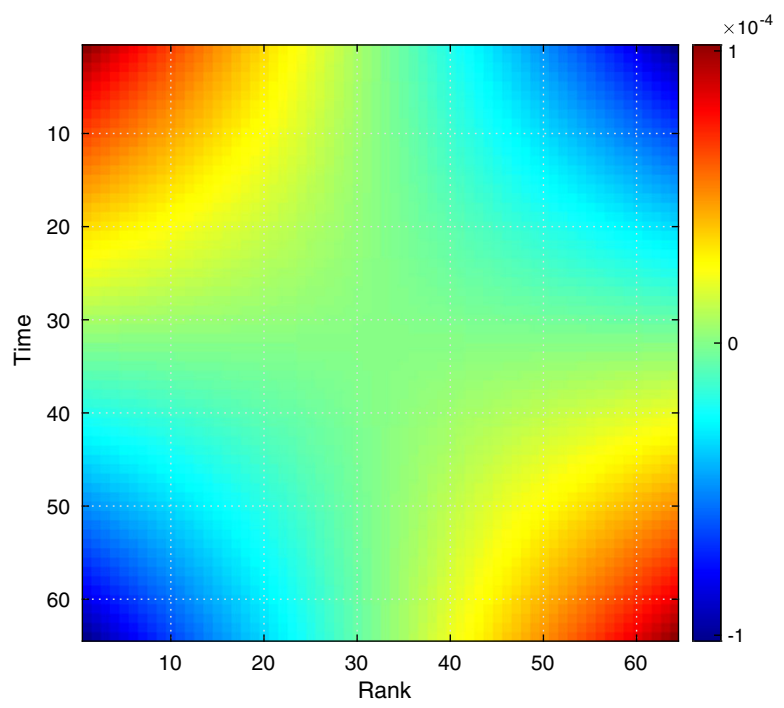

FIG. 18. $\langle Q\rangle$ as a filtered product of $P$. Here, the contracting row vector $\mathbf{m}^{\mathrm{T}}=\mathbf{1}^{\mathrm{T}} M$ is reshaped as a matrix to clarify its role in extracting a trend from $P$. 
[1] P. R. Bevington and D. K. Robinson, Data Reduction and Error Analysis for the Physical Sciences (McGraw-Hill, New York, 1992).

[2] S. M. Kay, Fundamentals of Statistical Signal Processing, Volume II: Detection Theory (Prentice, Upper Saddle River, NJ, 1998).

[3] T. A. Helble, G. R. Ierley, G. L. D’Spain, M. A. Roch, and J. A. Hildebrand, A Generalized Power-Law Detection Algorithm for Humpback Whale Vocalizations, J. Acoust. Soc. Am. 131, 2682 (2012).

[4] R. Lupton, Statistics in Theory and Practice (Princeton University Press, Princeton, NJ, 1993).

[5] F. Bardou, J.-P. Bouchaud, A. Aspect, and C. Cohen-Tannoudji, Lévy Statistics and Laser Cooling: How Rare Events Bring Atoms to Rest (Cambridge University Press, Cambridge, England, 2002).

[6] R. E. Benestad, How Often Can We Expect a Record Event?, Climate research 25, 3 (2003).

[7] R. E. Benestad, Record-Values, Non-stationarity Tests and Extreme Value Distributions, Global Planetary Change 44, 11 (2004).

[8] G. A. Meehl, C. Tebaldi, G. Walton, D. Easterling, and L. McDaniel, Relative Increase of Record High Maximum Temperatures Compared to Record Low Minimum Temperatures in the U.S., Geophys. Res. Lett. 36 (2009).

[9] A. Anderson and A. Kostinski, Reversible Record Breaking and Variability: Temperature Distributions Across the Globe, J. Appl. Meteorol. Clim. 49, 1681 (2010).

[10] D. Coumou and S. Rahmstorf, A Decade of Weather Extremes, Nature Climate Change 2, 491 (2012).

[11] Y. Edery, A. B. Kostinski, S. N. Majumdar, and B. Berkowitz, Record-Breaking Statistics for Random Walks in the Presence of Measurement Error and Noise, Phys. Rev. Lett. 110, 180602 (2013).

[12] W. J. Thompson and J. R. Macdonald, Correcting Parameter Bias Caused by Taking Logs of Exponential Data, Am. J. Phys. 59, 854 (1991).

[13] F. G. Foster and A. Stuart, Distribution-Free Tests in TimeSeries Based on the Breaking of Records Division of Research Techniques, London School of Economics, J. R. Stat. Soc., Ser. B 16, 1 (1954).

[14] The row index of $Q$ is a timelike coordinate. Its $N-1$ values lie at the midpoints of the original grid with $N$ points.

[15] The incomplete gamma function used here is 8.2.2 of the Digital Library of Mathematical Functions.

[16] A. C. Koivunen and A. B. Kostinski, The Feasibility of Data Whitening to Improve Performance of Weather Radar, J. Appl. Meteorology 38, 741 (1999).

[17] In higher dimensions, the first term of this decomposition has symmetry $B_{n}$, the hyperoctahedral group.

[18] B. Verberck, Symmetry-Adapted Fourier Series for the Wallpaper Groups, Symmetry 4, 379 (2012).

[19] As commented in Ref. [18], "A more formal approach for deriving minimal symmetry-adapted functions for the wallpaper groups involves group theory; each wallpaper group should be decomposed into irreducible representations." We note the wallpaper group $p 6 \mathrm{~mm}$ of graphene has a point group expansion from Eq. (14) in $D_{4}$ and $D_{2}$ only.

[20] For a square matrix populated by iid normal entries, the ensemble variances are $\sigma_{q_{(x, y)}^{\left(C_{1}\right)}}^{2}=1 / 4, \sigma_{q^{\left(D_{2}\right)}}^{2}=1 / 8$ as the matrix dimension tends to infinity, with the remainder apportioned in enigmatic proportion between $q^{\left(D_{4}\right)}$ and $q^{\left(R_{2}\right)}$.

[21] E. N. Lorenz, Empirical Orthogonal Functions and Statistical Weather Prediction (1956).

[22] The $x-y$ categorization used here breaks down at higher order when further bifurcations cause complex patterns, but these are not of practical concern.

[23] A. M. Yaglom, An Introduction to the Theory of Stationary Random Functions (Courier Corporation, Chelmsford, MA, 2004).

[24] For simplicity, we have omitted modes for spurious trends in variance of rank, which would symmetrize the table with a complementary entry in the third line.

[25] The magnitude of $\psi_{k}$ is arbitrary when this is done, but it has an upper bound that, if exceeded, induces negative elements in the resulting $P$.

[26] Note that $\delta \mu_{1}(x)$, with one inflection point, is not quite linear; hence, an exactly linear trend in the data maps onto an expansion in odd modes, though dominated by the first. Note that $\delta \mu_{k}(x)$ and $\delta r_{k}(x)$ curves, each as a set, are not orthogonal, in contrast to the group PCA modes.

[27] With reference to Table I, this mode pair $(13,14)$ is a bifurcation in the sequence, namely, the first for which the odd index of a mixed-mode planform exceeds unity. One conjectures that a similar inversion of rank and data mean is obtained for succeeding members of that bifurcation.

[28] The appearance of $\ln 2$ here suggests that the binary decision underlying ranking can play a role. This is reminiscent of $k T$ $\ln 2$ in the Landauer principle.

[29] From the expansion in Eq. (14) for Lugano, $Q^{\left(D_{4}\right)}$ accounts for $99.2 \%$ of the content of the $Q$ matrix in Fig. 2(b), the highest fraction for any station observed.

[30] B. Lehle, Analysis of Stochastic Time Series in the Presence of Strong Measurement Noise, Phys. Rev. E 83, 021113 (2011).

[31] M. B. Priestley, Spectral Analysis and Time Series (Academic Press, New York, 1981), pp. 120-121.

[32] D. B. Percival, A. T. Walden et al., Spectral Analysis for Physical Applications (Cambridge University Press, Cambridge, England, 1993), pp. 392-393.

[33] T. Scholz, F. Raischel, V. V. Lopes, B. Lehle, M. Wachter, J. Peinke, and P. G. Lind, Parameter-Free Resolution of the Superposition of Stochastic Signals, Phys. Lett. A 381, 194 (2017).

[34] One case, not shown in Fig. 8, yields no discernible departure from $\pi$ noise, and that is the first billion decimal digits of $\pi$. This holds for strings of $1,2,3$, and 4 digits after accounting for ties. Thus, $Q$ perceives digits of $\pi$ as iid noise, hence the name.

[35] A. Van der Ziel, Noise in Measurements (Wiley, New York, 1976).

[36] C. Bandt and B. Pompe, Permutation Entropy: A Natural Complexity Measure for Time Series, Phys. Rev. Lett. 88, 174102 (2002).

[37] O. A. Rosso, H. A. Larrondo, M. T. Martin, A. Plastino, and M. A. Fuentes, Distinguishing Noise from Chaos, Phys. Rev. Lett. 99, 154102 (2007). 
[38] P. Cvitanović, R. Artuso, R. Mainieri, G. Tanner, and G. Vattay, Chaos: Classical and Quantum (Niels Bohr Institute, Copenhagen, 2016).

[39] W. J. Wiscombe and J. W. Evans, Exponential-Sum Fitting of Radiative Transmission Functions, J. Comput. Phys. 24, 416 (1977).

[40] D. Landowne, B. Yuan, and K. L. Magleby, Exponential Sum-Fitting of Dwell-Time Distributions without Specifying Starting Parameters, Biophys. J. 104, 2383 (2013).

[41] D. P. Oleary and B. W. Rust, Variable Projection for Nonlinear Least Squares Problems, Comput. Optim. Applic. 54, 579 (2013).

[42] E. Yeramian and P. Claverie, Analysis of Multiexponential Functions without a Hypothesis as to the Number of Components, Nature (London) 326, 169 (1987).

[43] One downside compared with high-order regressions of linear least squares problems: The latter is solved by simple matrix inversion. Use of $Q_{\mathrm{rms}}$ gives a nonlinear minimization problem, though a robust one for all cases we have explored.

[44] Note a tentative parallel result for signal extraction of nonstationary variance, namely, $\delta \sigma(y)=\int^{y} d y^{\prime \prime} \int_{-1}^{1} d x^{\prime \prime} x^{\prime \prime} q\left(x^{\prime \prime}, y^{\prime \prime}\right)$. Applied to the data of Fig. 1, one finds a quadratic signal indicating the principal contribution is the first $R_{2}$ mode, $\psi_{6}$.

[45] We are indebted to Professor Peter Shearer of UCSD for the raw data from a forthcoming publication; P. M. Shearer and J. Buehler, Imaging Upper-Mantle Structure under
USArray Using Long-Period Reflection Seismology, JGR: Solid Earth (2019).

[46] G. Balkema and P. Embrechts, Linear Regression for Heavy Tails, Risks 6, 93 (2018).

[47] S. El Adlouni, T. B. M. J. Ouarda, X. Zhang, R. Roy, and B. Bobeé, Generalized Maximum Likelihood Estimators for the Nonstationary Generalized Extreme Value Model, Water Resour. Res. 43, W03410 (2007).

[48] Or, for that matter, $f_{1}\left(x ; c_{1}\right)+f_{2}(x ; c 2)$.

[49] X. Wang, X. Dang, H. Peng, and H. Zhang, The Theil-Sen Estimators in a Multiple Linear Regression Model, http:// home.olemiss.edu/ xdang/papers/MTSE.pdf.

[50] D. Slepian, Prolate Spheroidal Wave Functions, Fourier Analysis, and Uncertainty: The Discrete Case, Bell Syst. Tech. J. 57, 1371 (1978).

[51] D. J. Thomson, Spectrum Estimation and Harmonic Analysis, Proc. IEEE 70, 1055 (1982).

[52] For chaos, a nonvanishing ensemble mean of $Q$ is general even with rows of independent trials. The deterministic nature of such processes invalidates a result like Eq. (B1).

[53] See Supplemental Material at http://link.aps.org/ supplemental/10.1103/PhysRevX.9.031039 for two MATLAB programs for computing $P$ and $Q$, the latter simply encoding Eq. (1), which takes $\mathcal{O}\left(n_{T}^{4}\right)$ time.

[54] It is even more instructive to examine the eigenvectors and eigenvalues of $\mathbf{r m}$ reconstituted as a matrix. 